\title{
PoInTER: Constrained Progressive Text Generation via Insertion-based Generative Pre-training
}

\author{
Yizhe Zhang ${ }^{1 *}$ \\ Guoyin Wang ${ }^{2 * \dagger}$ \\ Chunyuan $\mathbf{L i}^{1}$ \\ Zhe Gan ${ }^{1}$ \\ Chris Brockett ${ }^{1}$ \\ Bill Dolan ${ }^{1}$ \\ ${ }^{1}$ Microsoft Research, Redmond, WA, USA \\ ${ }^{2}$ Amazon Alexa AI, Seattle, WA, USA \\ \{yizzhang, chunyl, zhe.gan, chrisbkt,billdol\}@microsoft.com, guoyiwan@amazon.com
}

\begin{abstract}
Large-scale pre-trained language models, such as BERT and GPT-2, have achieved excellent performance in language representation learning and free-form text generation. However, these models cannot be directly employed to generate text under specified lexical constraints. To address this challenge, we present POINTER $^{1}$, a simple yet novel insertion-based approach for hard-constrained text generation. The proposed method operates by progressively inserting new tokens between existing tokens in a parallel manner. This procedure is recursively applied until a sequence is completed. The resulting coarse-to-fine hierarchy makes the generation process intuitive and interpretable. We pre-train our model with the proposed progressive insertion-based objective on a 12GB Wikipedia dataset, and finetune it on downstream hard-constrained generation tasks. Non-autoregressive decoding yields an empirically logarithmic time complexity during inference time. Experimental results on both News and Yelp datasets demonstrate that POINTER achieves state-of-the-art performance on constrained text generation. We released the pre-trained models and the source code to facilitate future research ${ }^{2}$.
\end{abstract}

\section{Introduction}

Real-world editorial assistant applications must often generate text under specified lexical constraints, for example, convert a meeting note with key phrases into a concrete meeting summary, recast a user-input search query as a fluent sentence, generate a conversational response using grounding facts (Mou et al., 2016), or create a story using a pre-specified set of keywords (Fan et al., 2018; Yao et al., 2019; Donahue et al., 2020).

\footnotetext{
${ }^{*}$ These authors contributed equally to this work.

${ }^{\dagger}$ Work was done while Guoyin was at Microsoft.

${ }^{1}$ PrOgressive INsertion-based TransformER

${ }^{2}$ https://github.com/dreasysnail/POINTER
}

Generating text under specific lexical constraints is challenging. Constrained text generation broadly falls into two categories, depending on whether inclusion of specified keywords in the output is mandatory. In soft-constrained generation (Qin et al., 2019; Tang et al., 2019), keyword-text pairs are typically first constructed (sometimes along with other conditioning information), and a conditional text generation model is trained to capture their co-occurrence, so that the model learns to incorporate the constrained keywords into the generated text. While soft-constrained models are easy to design, even remedied by soft enforcing algorithms such as attention and copy mechanisms (Bahdanau et al., 2015; Gu et al., 2016; Chen et al., 2019a), keywords are still apt to be lost during generation, especially when multiple weakly correlated keywords must be included.

Hard-constrained generation (Hokamp and Liu, 2017; Post and Vilar, 2018; Hu et al., 2019; Miao et al., 2019; Welleck et al., 2019), on the other hand, requires that all the lexical constraints be present in the output sentence. This approach typically involves sophisticated design of network architectures. Hokamp and Liu (2017) construct a lexical-constrained grid beam search decoding algorithm to incorporate constraints. However, $\mathrm{Hu}$ et al. (2019) observe that a naive implementation of this algorithm has a high running time complexity. Miao et al. (2019) introduces a sampling-based conditional generation method, where the constraints are first placed in a template, then words in a random position are either inserted, deleted or updated under a Metropolis-Hastings-like scheme. However, individually sampling each token results in slow convergence, as the joint distribution of all the tokens in a sentence is highly correlated. Welleck et al. (2019) propose a tree-based text generation scheme, where a token is first generated in an arbitrary position, and then the model recursively 


\begin{tabular}{|c|l|}
\hline Stage & Generated text sequence \\
$0\left(X^{0}\right)$ & sources sees structure perfectly \\
$1\left(X^{1}\right)$ & sources company sees change structure perfectly legal \\
$2\left(X^{2}\right)$ & sources suggested company sees reason change tax structure which perfectly legal . \\
$3\left(X^{3}\right)$ & my sources have suggested the company sees no reason to change its tax structure, which are perfectly legal . \\
$4\left(X^{4}\right)$ & my sources have suggested the company sees no reason to change its tax structure, which are perfectly legal . \\
\hline
\end{tabular}

Table 1: Example of the progressive generation process with multiple stages from the POINTER model. Words in blue indicate newly generated words at the current stage. $X^{i}$ denotes the generated partial sentence at Stage $i$. $X^{4}$ and $X^{3}$ are the same indicates the end of the generation process. Interestingly, our method allows informative words (e.g., company, change) generated before the non-informative words (e.g., the, to) generated at the end.

generates words to its left and right, yielding a binary tree. However, the constructed tree may not reflect the progressive hierarchy/granularity from high-level concepts to low-level details. Further, the time complexity of generating a sentence is $\mathcal{O}(n)$, like standard auto-regressive methods.

Motivated by the above, we propose a novel nonautoregressive model for hard-constrained text generation, called Pointer (PrOgressive INsertionbased TransformER). As illustrated in Table 1, generation of words in POINTER is progressive, and iterative. Given lexical constraints, POINTER first generates high-level words (e.g., nouns, verbs and adjectives) that bridge the keyword constraints, then these words are used as pivoting points at which to insert details of finer granularity. This process iterates until a sentence is finally completed by adding the least informative words (typically pronouns and prepositions).

Due to the resemblance to the masked language modeling (MLM) objective, BERT(Devlin et al., 2019) can be naturally utilized for initialization. Further, we perform large-scale pre-training on a large Wikipedia corpus to obtain a pre-trained POINTER model that which can be readily finetuned on specific downstream tasks.

The main contributions of this paper are summarized as follows. (i) We present POINTER, a novel insertion-based Transformer model for hardconstrained text generation. Compared with previous work, POINTER allows long-term control over generation due to the top-down progressive structure, and enjoys a significant reduction over emperical time complexity from $\mathcal{O}(n)$ to $\mathcal{O}(\log n)$ at best. (ii) Large-scale pre-training and novel beam search algorithms are proposed to further boost performance. (iii) We develop a novel beam search algorithm customized to our approach, further improving the generation quality. (iv) Experiments on several datasets across different domains (including News and Yelp) demonstrates the superiority of POINTER over strong baselines. Our approach is simple to understand and implement, yet powerful, and can be leveraged as a building block for future research.

\section{Related Work}

Language Model Pre-training Large-scale pretrained language models, such as BERT (Devlin et al., 2019), RoBERTa (Liu et al., 2019), XLNet (Yang et al., 2019), Text-to-text Transformer (Raffel et al., 2019) and ELECTRA (Clark et al., 2020), have achieved great success on natural language understanding benchmarks. GPT2 (Radford et al., 2018) first demonstrates great potential for leveraging Transformer models in generating realistic text. MASS (Song et al., 2019) and BART (Lewis et al., 2019) propose methods for sequence-to-sequence pre-training. UniLM (Dong et al., 2019) unifies the generation and understanding tasks within a single pre-training scheme. DialoGPT (Zhang et al., 2020) and MEENA (Adiwardana et al., 2020) focus on opendomain conversations. CTRL (Keskar et al., 2019) and Grover (Zellers et al., 2019) guide text generation with pre-defined control codes. In addition, recent work has also investigated how to leverage BERT for conditional text generation (Chen et al., 2019b; Mansimov et al., 2019; Li et al., 2020). To the best of our knowledge, ours is the first largescale pre-training work for hard-constrained text generation.

Non-autoregressive Generation Many attempts have been made to use non-autoregressive models for text generation tasks. For neural machine translation, the promise of such methods mostly lies in their decoding efficiency. For example, Gu et al. (2018) employs a non-autoregressive decoder that generates all the tokens simultaneously. Generation can be further refined with a post-processing step to remedy the conditional independence of the parallel decoding process (Lee et al., 2018; Ghazvininejad et al., 2019; Ma et al., 2019; Sun 
et al., 2019; Kasai et al., 2020). Deconvolutional decoders (Zhang et al., 2017; Wu et al., 2019) have also been studied for title generation and machine translation. The Insertion Transformer (Stern et al., 2019; Gu et al., 2019; Chan et al., 2019) is a partially autoregressive model that predicts both insertion positions and tokens, and is trained to maximize the entropy over all valid insertions, providing fast inference while maintaining good performance. Our POINTER model hybridizes the BERT and Insertion Transformer models, inheriting the advantages of both, and generates text in a progressive coarse-to-fine manner.

\section{Method}

\subsection{Model Overview}

Let $X=\left\{x_{0}, x_{1}, \cdots, x_{T}\right\}$ denote a sequence of discrete tokens, where each token $x_{t} \in V$, and $V$ is a finite vocabulary set. For the hard-constrained text generation task, the goal is to generate a complete text sequence $X$, given a set of key words $\hat{X}$ as constraints, where the key words have to be exactly included in the final generated sequence with the same order.

Let us denote the lexical constraints as $X^{0}=\hat{X}$. The generation procedure of our method can be formulated as a (progressive) sequence of $K$ stages: $S=\left\{X^{0}, X^{1}, \cdots, X^{K-1}, X^{K}\right\}$, such that for each $k \in\{1, \ldots, K\}, X^{k-1}$ is a sub-sequence of $X^{k}$. The following stage can be perceived as a finer-resolution text sequence compared to the preceding stage. $X^{K}$ is the final generation, under the condition that the iterative procedure is converged (i.e., $X^{K-1}=X^{K}$ ).

Table 1 shows an example of our progressive text generation process. Starting from the lexical constraints $\left(X_{0}\right)$, at each stage, the algorithm inserts tokens progressively to formulate the target sequence. At each step, at most one new token can be generated between two existing tokens. Formally, we propose to factorize the distribution according to the importance (defined later) of each token:

$$
p(X)=p\left(X^{0}\right) \prod_{k=1}^{K} p\left(X^{k} \mid X^{k-1}\right)
$$

where $p\left(X^{k} \mid X^{k-1}\right)=\prod_{x \in X^{k}-X^{k-1}} p\left(x \mid X^{k-1}\right)$. The more important tokens that form the skeleton of the sentence, such as nouns and verbs, appear in earlier stages, and the auxiliary tokens, such as articles and prepositions, are generated at the later stages. In contrast, the autoregressive model factorizes the joint distribution of $X$ in a standard left-toright manner, i.e., $p(X)=p\left(x_{0}\right) \prod_{t=1}^{T} p\left(x_{t} \mid x_{<t}\right)$, ignoring the word importance. Though the Insertion Transformer (Stern et al., 2019) attempts to implement the progressive generation agenda in (1), it does not directly address how to train the model to generate important tokens first.

\subsection{Data Preparation}

Designing a loss function so that $(i)$ generating an important token first and (ii) generating more tokens at each stage that would yield a lower loss would be complicated. Instead, we prepare data in a form that eases model training.

The construction of data-instance pairs reverses the generation process. We construct pairs of text sequences at adjacent stages, i.e., $\left(X^{k-1}, X^{k}\right)$, as the model input. Therefore, each training instance $X$ is broken into a consecutive series of pairs: $\left(X^{0}, X^{1}\right), \cdots,\left(X^{K-1}, X^{K}\right)$, where $K$ is the number of such pairs. At each iteration, the algorithm masks out a proportion of existing tokens $X^{k}$ to yield a sub-sequence $X^{k-1}$, creating a training instance pair $\left(X^{k-1}, X^{k}\right)$. This procedure is iterated until only less than $c$ ( $c$ is small) tokens are left.

Two properties are desired when constructing data instances: (i) important tokens should appear in an earlier stage, so that the generation follows a progressive manner; ( $i i)$ the number of stages $K$ is small, thus the generation is fast at inference time.

Token Importance Scoring We consider three different schemes to assess the importance score of a token: term frequency-inverse document frequency (TF-IDF), part-of-speech (POS) tagging, and Yet-Another-Keyword-Extractor (YAKE) (Campos et al., 2018, 2020). The TF-IDF score provides the uniqueness and local enrichment evaluation of a token at a corpus level. POS tagging indicates the role of a token at a sequence level. We explicitly assign noun or verb tokens a higher POS tagging score than tokens from other categories. YAKE is a commonly used unsupervised automatic keyword extraction method that relies on statistical features extracted from single documents to select the most important keywords (Campos et al., 2020). YAKE is good at extracting common key words, but relatively weak at extracting special nouns (e.g., names), and does not provide any importance level for non-keyword tokens. Therefore, we combine the above three metrics for token importance scor- 
ing. Specifically, the overall score $\alpha_{t}$ of a token $x_{t}$ is defined as $\alpha_{t}=\alpha_{t}^{\mathrm{TF}-\mathrm{IDF}}+\alpha_{t}^{\mathrm{POS}}+\alpha_{t}^{\mathrm{YAKE}}$, where $\alpha_{t}^{\mathrm{TF}-\mathrm{IDF}}, \alpha_{t}^{\mathrm{POS}}$ and $\alpha_{t}^{\mathrm{YAKE}}$ represent the TF-IDF, POS tagging and YAKE scores (each is rescaled to $[0,1])$, respectively.

Additionally, stop words are manually assigned a low importance score. If a token appears several times in a sequence, the latter occurrences are assigned a decayed importance score to prevent the model from generating the same token multiple times in one step at inference time. We note that our choice of components of the importance score is heuristic. It would be better to obtain an unbiased/oracle assessment of importance, which we leave for future work.

DP-based Data Pair Construction Since we leverage the Insertion-based Transformer, which allows at most one new token to be generated between each two existing tokens, sentence length at most doubles at each iteration. Consequently, the optimal number of iterations $K$ is $\log (T)$, where $T$ is the length of the sequence. Therefore, generation efficiency can be optimized by encouraging more tokens to be discarded during each masking step when preparing the data. However, masking positional interleaving tokens ignores token importance, and thus loses the property of progressive planning from high-level concepts to low-level details at inference time. In practice, sequences generated by such an approach can be less semantically consistent as less important tokens occasionally steer generation towards random content.

We design an approach to mask the sequence by considering both token importance and efficiency using dynamic programming (DP). To accommodate the nature of insertion-based generation, the masking procedure is under the constraint that no consecutive tokens can be masked at the same stage. Under such a condition, we score each token and select a subset of tokens that add up to the highest score (all scores are positive). This allows the algorithm to adaptively choose as many high scored tokens as possible to mask.

Formally, as an integer linear programming problem (Richards and How, 2002), the objective is to find an optimal masking pattern $\Phi=$ $\left\{\phi_{1}, \cdots, \phi_{T}\right\}$, where $\phi_{t} \in\{0,1\}$, and $\phi_{t}=1$ represents discarding the corresponding token $x_{t}$, and $\phi_{t}=0$ indicates $x_{t}$ remains. For a sequence $X^{\prime}$,

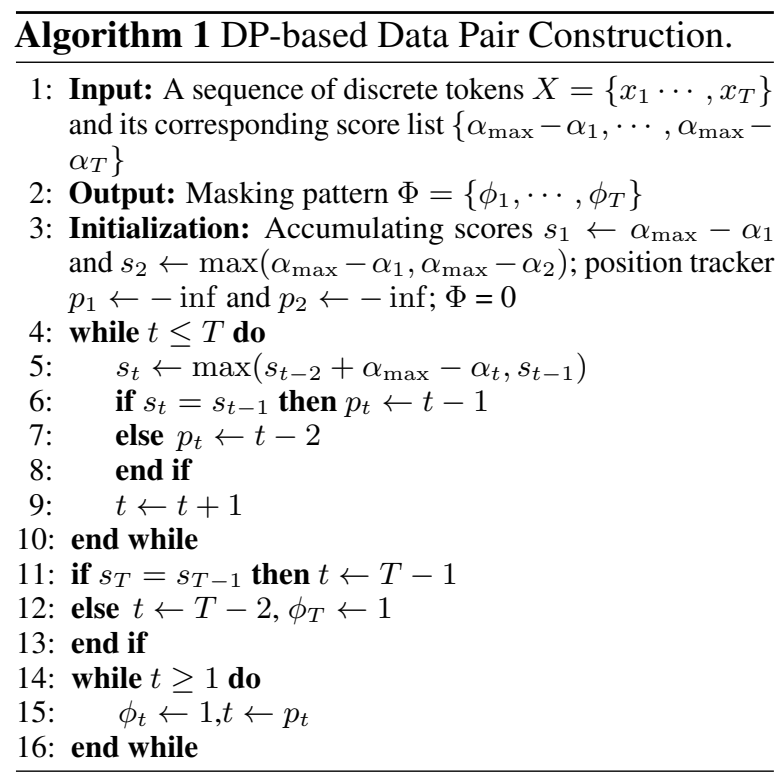

the objective can be formulated as:

$$
\begin{array}{r}
\max \sum_{t=1}^{T} \phi_{t}\left(\alpha_{\max }-\alpha_{t}\right), \\
\text { s.t. } \quad \phi_{t} \phi_{t+1} \neq 1, \forall t
\end{array}
$$

where $\alpha_{\max }=\max _{t}\left\{\alpha_{t}\right\}$. Though solving Eq. (2) is computationally expensive, one can resort to an analogous problem for a solution, the socalled House Robbery Problem, a variant of Maximum Subarray Problem (Bentley, 1984), where a professional burglar plans to rob houses along a street and tries to maximize the outcome, but cannot break into two adjacent houses without triggering an alarm. This can be solved using dynamic programming (Bellman, 1954) (also known as Kadane's algorithm (Gries, 1982)) as shown in Algorithm 1.

\subsection{Model Training}

Stage-wise Insertion Prediction With all the data-instance pairs $\left(X^{k-1}, X^{k}\right)$ created as described above as the model input, we optimize the following objective:

$$
\begin{aligned}
& \mathcal{L}=-\log p\left(X^{k} \mid X^{k-1}\right) \\
& =-\sum_{x \in X^{+}} \log p\left(x \mid \Phi^{k-1}, X^{k-1}\right) p\left(\Phi^{k-1} \mid X^{k-1}\right),
\end{aligned}
$$

where $X^{+} \triangleq X^{k}-X^{k-1}$, and $\Phi^{k-1}$ denotes an indicator vector in the $k$-th stage, representing whether an insertion operation is applied in a slot.

As illustrated in Figure 1, while the MLM objective in BERT only predicts the token of a masked 


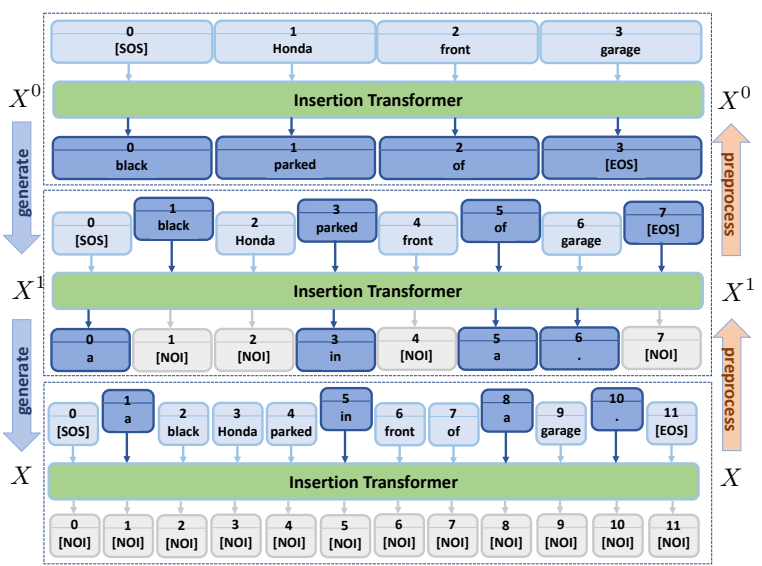

Figure 1: Illustration of the generation process $\left(X^{0} \rightarrow\right.$ $X)$ of the proposed POINTER model. At each stage, the Insertion Transformer module generates either a regular token or a special [NOI] token for each gap between two existing tokens. The generation stops when all the gaps predict [NOI]. The data preparation process reverses the above generative process.

placeholder, our objective comprises both $(i)$ likelihood of an insertion indicator for each slot (between two existing tokens), and (ii) the likelihood of each new token conditioning on the activated slot. To handle this case, we expand the vocabulary with a special no-insertion token [NOI]. During inference time, the model can predict either a token from the vocabulary to insert, or an [NOI] token indicating no new token will be inserted at a certain slot at the current stage. By utilizing this special token, the two objectives are merged. Note that the same insertion transformer module is re-used at different stages. We empirically observed that the model can learn to insert different words at different stages; it presumably learns from the completion level (how discontinuous the context is) of the current context sequence to roughly estimate the progress up to that point.

During inference time, once in a stage $\left(X^{k}\right)$, all the slots predict [NOI] for the next stage, the generation procedure is converged and $X^{k}$ is the final output sequence. Note that to account for this final stage $X^{k}$, during data preparation we incorporate an $(X, N)$ pair for each sentence in the training data, where $N$ denotes a sequence of [NOI] with the same length of $X$. To enable the model to insert at the beginning and end of the sequence, an [SOS] token and an [EOS] token are added in the beginning and at the end of each sentence, respectively.

In light of the similarity with the MLM objective, we use BERT model to initialize the Insertion Transformer module.
Large-scale Pre-training In order to provide a general large-scale pretrained model that can benefit various downstream tasks with fine-tuning, we train a model on the massive publicly available English Wiki dataset, which covers a wide range of topics. The Wiki dataset is first preprocessed according to Sec. 3.2. We then initialize the model with BERT, and perform model training on the processed data using our training objective (3). After pre-training, the model can be used to generate an appropriate sentence with open-domain keyword constraints, in a tone that represents the Wiki style. In order to adapt the pre-trained model to a new domain (e.g., News and Yelp reviews), the pre-trained model is further fine-tuned on new datasets, which empirically demonstrates better performance than training the model on the target domain alone.

\subsection{Inference}

During inference time, starting from the given lexical constraint $X^{0}$, the proposed model generates text stage-by-stage using greedy search or top-K sampling (Fan et al., 2018), by applying the Insertion Transformer module repeatedly until no additional token is generated. If a [NOI] token is generated, it is deleted at the next round.

Inner-Layer Beam Search According to (3), all new tokens are simultaneously generated based on the existing tokens at the previous stage. Despite of being fully parallel, like BERT (Yang et al., 2019) and NAT (Ghazvininejad et al., 2019; Kasai et al., 2020) this approach suffers from a conditional independence problem in which the predicted tokens are conditional-independently generated and are agnostic of each other. This can result in generating repeating or inconsistent new tokens at each generation round. ${ }^{3}$

To address this weak-dependency issue, we perform a modified beam search algorithm for decoding. Specifically, at stage $k$, suppose the existing tokens from last stage are $X^{k-1}=\left\{x_{1}^{k-1}, \cdots, x_{T_{k-1}}^{k-1}\right\}$, where $T_{k-1}$ is the length of $X^{k-1}$. For predicting next stage $X^{k}$, there will be $T_{k-1}$ available slots. A naive approach to perform beam search would be to maintain a priority queue of top $B$ candidate token series predictions when moving from the leftmost slot to the rightmost slot. At the $t$-th move, the priority queue contains top $B$ sequences

\footnotetext{
${ }^{3}$ For example, from an existing token "and", the model generates "clean and clean".
} 
for existing predicted tokens: $\left(s_{1}^{(b)}, \cdots, s_{t-1}^{(b)}\right)$, where $s_{i}^{(b)}$ denotes the predicted token for the $i$-th slot in the $b$-th $(b \in\{1, \cdots, B\})$ sequence. The model then evaluates the likelihood of each item (including [NOI]) in the vocabulary for the slot $s_{t}$, by computing the likelihood of $\left(s_{1}^{(b)}, x_{1}^{k-1}, \cdots, s_{t-1}^{(b)}, x_{t-1}^{k-1}, s_{t}, x_{t}^{k-1},[\mathrm{NOI}], \cdots\right.$, [NOI], $x_{T_{k-1}}^{k-1}$ ). This is followed by a ranking step to select the top $B$ most likely series among the $V B$ series to grow. However, such a naive approach is expensive, as the runtime complexity takes $\mathcal{O}(T B V)$ evaluations.

Instead, we approximate the search by constraining it in a narrow band. We design a customized beam search algorithm for our model, called innerlayer beam search (ILBS). This method applies an approximate local beam search at each iteration to find the optimal stage-wise decoding. At the $t$-th slot, ILBS first generates top $B$ token candidates by applying one evaluation step based on existing generation. Prediction is limited to these top $B$ token candidates, and thus the beam search procedure as described above is applied on the narrow band of $B$ instead of the full vocabulary $V$. This reduces the computation to $\mathcal{O}\left(T B^{2}\right)$.

\section{Experiments}

We evaluate the POINTER model on constrained text generation over News and Yelp datasets. Details of the datasets and experimental results are provided in the following sub-sections. The pretrained models and the source code are available at Github ${ }^{4}$.

\subsection{Experimental Setup}

Datasets and Pre-processing We evaluate our model on two datasets. The EMNLP2017 WMT News dataset ${ }^{5}$ contains 268,586 sentences, and we randomly pick 10k sentences as the validation set, and $1 \mathrm{k}$ sentences as the test set. The Yelp English review dataset is from Cho et al. (2018), which contains $160 \mathrm{k}$ training examples, $10 \mathrm{k}$ validation examples and $1 \mathrm{k}$ test examples. These two datasets vary in sentence length and domain, enabling the assessment of our model in different scenarios.

The English Wikipedia dataset we used for pretraining is first pre-processed into a set of natural sentences, with maximum sequence length of 64 tokens, which results in 1.99 million sentences for

\footnotetext{
${ }^{4}$ https://github.com/dreasysnail/POINTER

${ }^{5}$ http: //www. statmt.org/wmt17/
}

model training in total (12.6 GB raw text). On average, each sentence contains 27.4 tokens.

For inference, we extract the testing lexical constraints for all the compared methods using the 3rd party extracting tool $\mathrm{YAKE}^{6}$. The maximum length of the lexical constraints we used for News and Yelp is set to 4 and 7, respectively, to account the average length for News $\left(27.9 \approx 4 \times 2^{3}\right)$ and Yelp ( $50.3 \approx 7 \times 2^{3}$ ), as we would hope the generation can be done within 4 stages.

Baselines We compare our model with two stateof-the-art methods for hard-constrained text generation: ( $i$ ) Non-Monotonic Sequential Text Generation (NMSTG) (Welleck et al., 2019), and (ii) Constrained Sentence Generation by MetropolisHastings Sampling (CGMH) (Miao et al., 2019). We also compared with an autoregressive softconstraint baseline(Gao et al., 2020). Note that the Insertion Transformer (Stern et al., 2019) focuses on machine translation rather than hard-constrained generation task, and therefore is not considered for comparison. Other methods based on grid beam search typically have long inference time, and they only operate on the inference stage; these are also excluded from comparison. For all compared system, we use the default settings suggested by the authors, the models are trained until the evaluation loss does not decrease. More details are provided in the Appendix.

Experiment Setups We employ the tokenizer and model architecture from BERT-base and BERTlarge models for all the tasks. BERT models are used as our model initialization. Each model is trained until the validation loss is no longer decreasing. We use a learning rate of $3 e-5$ without any warming-up schedule for all the training procedures. The optimization algorithm is Adam (Kingma and $\mathrm{Ba}, 2015$ ). We pre-train our model on the Wiki dataset for 2-4 epochs, and fine-tune on the News and Yelp datasets for around 10 epochs.

Evaluation Metrics Following Zhang et al. (2020), we perform automatic evaluation using commonly adopted text generation metrics, including BLEU (Papineni et al., 2002), METEOR (Lavie and Agarwal, 2007), and NIST (Doddington, 2002). Following (Kann et al., 2018), to assess the coherence of generated sentences, we also report the perplexity over the test set using pre-trained GPT-2

\footnotetext{
${ }^{6}$ https://github.com/LIAAD/yake
} 


\begin{tabular}{|c|c|c|c|c|c|c|c|c|c|c|}
\hline \multirow{2}{*}{$\begin{array}{r}\text { News dataset } \\
\text { Method }\end{array}$} & \multicolumn{2}{|c|}{ NIST } & \multicolumn{2}{|c|}{ BLEU } & \multirow[t]{2}{*}{ METEOR } & \multirow{2}{*}{$\begin{array}{r}\text { Entropy } \\
\text { E-4 }\end{array}$} & \multicolumn{2}{|c|}{ Dist } & \multirow[t]{2}{*}{ PPL. } & \multirow[t]{2}{*}{ Avg. Len. } \\
\hline & $\mathrm{N}-2$ & $\mathrm{~N}-4$ & B-2 & B-4 & & & D-1 & D-2 & & \\
\hline CGMH & 1.60 & 1.61 & $7.09 \%$ & $1.61 \%$ & $12.55 \%$ & 9.32 & $16.60 \%$ & $70.55 \%$ & 189.1 & 14.29 \\
\hline NMSTG & 2.70 & 2.70 & $10.67 \%$ & $1.58 \%$ & $13.56 \%$ & 10.10 & $11.09 \%$ & $65.96 \%$ & 171.0 & 27.85 \\
\hline Greedy (base) & 2.90 & 2.80 & $12.13 \%$ & $1.63 \%$ & $15.66 \%$ & 10.41 & $5.89 \%$ & $39.42 \%$ & 97.1 & 47.40 \\
\hline Greedy (+Wiki,base) & 3.04 & 3.06 & $13.01 \%$ & $2.51 \%$ & $16.38 \%$ & 10.22 & $11.10 \%$ & $57.78 \%$ & 56.7 & 31.32 \\
\hline ILBS (+Wiki,base) & 3.20 & 3.22 & $14.00 \%$ & $2.99 \%$ & $15.71 \%$ & 9.86 & $13.17 \%$ & $61.22 \%$ & 66.4 & 22.59 \\
\hline Greedy (+Wiki, large) & 3.28 & 3.30 & $14.04 \%$ & $3.04 \%$ & $15.90 \%$ & 10.09 & $12.23 \%$ & $60.86 \%$ & 54.7 & 27.99 \\
\hline Human oracle & - & - & - & - & - & 10.05 & $11.80 \%$ & $62.44 \%$ & 47.4 & 27.85 \\
\hline \multirow{2}{*}{$\begin{array}{r}\text { Yelp dataset } \\
\text { Method }\end{array}$} & \multicolumn{2}{|c|}{ NIST } & \multicolumn{2}{|c|}{ BLEU } & METEOR & Entropy & \multicolumn{2}{|c|}{ Dist } & PPL. & Avg. Len. \\
\hline & $\mathrm{N}-2$ & $\mathrm{~N}-4$ & B-2 & B-4 & & E-4 & D-1 & D-2 & & \\
\hline $\mathrm{CGMH}$ & 0.50 & 0.51 & $4.53 \%$ & $1.45 \%$ & $11.87 \%$ & 9.48 & $12.18 \%$ & $57.10 \%$ & 207.2 & 16.70 \\
\hline NMSTG & 1.11 & 1.12 & $10.06 \%$ & $1.92 \%$ & $13.88 \%$ & 10.09 & $8.39 \%$ & $50.80 \%$ & 326.4 & 27.92 \\
\hline Greedy (base) & 2.15 & 2.15 & $11.48 \%$ & $2.16 \%$ & $17.12 \%$ & 11.00 & $4.19 \%$ & $31.42 \%$ & 99.5 & 87.30 \\
\hline Greedy (+Wiki,base) & 3.27 & 3.30 & $15.63 \%$ & $3.32 \%$ & $16.14 \%$ & 10.64 & $7.51 \%$ & $46.12 \%$ & 71.9 & 48.22 \\
\hline ILBS (+Wiki,base) & 3.34 & 3.38 & $16.68 \%$ & $3.65 \%$ & $15.57 \%$ & 10.44 & $9.43 \%$ & $50.66 \%$ & 61.0 & 35.18 \\
\hline Greedy (+Wiki, large) & 3.49 & 3.53 & $16.78 \%$ & $3.79 \%$ & $16.69 \%$ & 10.56 & $6.94 \%$ & $41.2 \%$ & 55.5 & 48.05 \\
\hline Human oracle & - & - & - & - & - & 10.70 & $10.67 \%$ & $52.57 \%$ & 55.4 & 50.36 \\
\hline
\end{tabular}

Table 2: Automatic evaluation results on the News (upper) and Yelp (lower) dataset. ILBS denotes beam search. "+Wiki" denotes fine-tuning on the Wiki-pretrained model. "base/large" represents the greedy generation from a based(110M)/large(340M) model. "Human" represents the held-out human reference.

medium (large) model ${ }^{7}$. We use Entropy (Zhang et al., 2018) and Dist-n (Li et al., 2016) to evaluate lexical diversity.

\begin{tabular}{l|l}
\hline Keywords & estate pay stay policy \\
\hline CGMH & $\begin{array}{l}\text { an economic estate developer that could pay } \\
\text { for it is that a stay policy . }\end{array}$ \\
\hline NMSTG & $\begin{array}{l}\text { as estate owners, they cannot pay for house- } \\
\text { holds for hundreds of middle - income property } \\
\text {, buyers stay in retail policy . }\end{array}$ \\
\hline $\begin{array}{l}\text { POINTER } \\
\text { (Greedy, } \\
\text { base) }\end{array}$ & $\begin{array}{l}\text { if you buy new buildings from real estate com- } \\
\text { and stay with the policy for financial reasons . }\end{array}$ \\
\hline $\begin{array}{l}\text { POINTER } \\
\text { (ILBS, } \\
\text { base) }\end{array}$ & $\begin{array}{l}\text { but no matter what foreign buyers do , real } \\
\text { estate agents will have to pay a small fee to } \\
\text { stay consistent with the policy . }\end{array}$ \\
\hline $\begin{array}{l}\text { POINTER } \\
\text { (Greedy, }\end{array}$ & $\begin{array}{l}\text { but it would also be required for estate agents } \\
\text { who must pay a larger amount of cash but stay } \\
\text { with the same policy for all other assets . }\end{array}$ \\
\hline
\end{tabular}

Table 3: Generated examples from the News dataset.

\subsection{Experimental Results}

News Generation We first conduct experiments on the News dataset to generate sentences from 4 lexical constraints. Quantitative results are summarized in Table 2 (upper). Some qualitative examples including the progressive generations at each stage are provided in Table 3 and Appendix B. POINTER is able to take full advantage of BERT initialization and Wiki pre-training to improve relevance scores (NIST, BLEU and METEOR). Lever-

\footnotetext{
${ }^{7}$ https://github.com/openai/gpt-2
}

\begin{tabular}{l|l}
\hline Keywords & joint great food great drinks greater staff \\
\hline CGMH & $\begin{array}{l}\text { very cool joint with great food , great drinks } \\
\text { and even greater staff . ! }\end{array}$ \\
\hline NMSTG & $\begin{array}{l}\text { awesome joint . great service. great food } \\
\text { great drinks. good to greater and great staff! }\end{array}$ \\
\hline $\begin{array}{l}\text { POINTER } \\
\text { (Greedy, }\end{array}$ & $\begin{array}{l}\text { my favorite local joint around old town. great } \\
\text { atmosphere, amazing food, delicious and deli- } \\
\text { base) } \\
\text { cous coffee, great wine selection and delicious } \\
\text { tio space and energetic front desk staff. }\end{array}$ \\
\hline $\begin{array}{l}\text { POINTER pa- } \\
\text { (ILBS, } \\
\text { base) }\end{array}$ & $\begin{array}{l}\text { the best breakfast joint in charlotte . great ser- } \\
\text { vice and amazing food . they have great selec- } \\
\text { tion of drinks that suits the greater aesthetic } \\
\text { of the staff . }\end{array}$ \\
\hline $\begin{array}{l}\text { POINTER } \\
\text { (Greedy, }\end{array}$ & $\begin{array}{l}\text { this is the new modern breakfast joint to be } \\
\text { found around the area . great atmosphere, cen- } \\
\text { tral location and excellent food . nice variety of } \\
\text { Large) } \\
\text { gections . great selection of local craft beers } \\
\text { greater price . very friendly patio and fun staff } \\
\text { love it ! }\end{array}$ \\
\hline
\end{tabular}

Table 4: Generated examples from the Yelp dataset. aging the ILBS or using a larger model further improves most automatic metrics we evaluated ${ }^{8}$. For diversity scores, as CGMH is a sampling-based method in nature, it achieves the highest Dist-n scores (even surpasses human score). We observed that the length of generated sentences, the diversity scores and the GPT-2 perplexity from POINTER are close to human oracle.

Yelp Generation We further evaluate our method

\footnotetext{
${ }^{8}$ The ILBS for larger models performs similarly to greedy decoding, and thus is omitted from comparison
} 


\begin{tabular}{|c|c|c|c|c|c|c|c|c|c|}
\hline \multicolumn{10}{|c|}{ Semantics: $A$ and $B$, which is more semantically meaningful and consistent? } \\
\hline \multicolumn{5}{|c|}{ News dataset } & \multicolumn{5}{|c|}{ Yelp dataset } \\
\hline \multicolumn{2}{|c|}{ System A } & Neutral & \multicolumn{2}{|c|}{ System B } & \multicolumn{2}{|c|}{ System A } & \multirow{3}{*}{$\begin{array}{c}\text { Neutral } \\
17.3 \% \\
23.0 \%\end{array}$} & \multicolumn{2}{|c|}{ System B } \\
\hline POINTER(base) & $60.9 \%$ & $17.4 \%$ & $21.8 \%$ & CGMH & PoInter(base) & $\mathbf{5 9 . 8 \%}$ & & $23.0 \%$ & CGMH \\
\hline POINTER(base) & $\mathbf{5 5 . 2 \%}$ & $21.7 \%$ & $23.1 \%$ & NMSTG & POINTER(base) & $57.5 \%$ & & $19.6 \%$ & NMSTG \\
\hline Pointer(base) & $21.7 \%$ & $21.4 \%$ & $\mathbf{5 6 . 9} \%$ & Human & Pointer(base) & $26.8 \%$ & $25.9 \%$ & $47.3 \%$ & Human \\
\hline \multicolumn{10}{|c|}{ Fluency: $A$ and $B$, which is more grammatical and fluent? } \\
\hline \multicolumn{5}{|c|}{ News dataset } & \multicolumn{5}{|c|}{ Yelp dataset } \\
\hline \multicolumn{2}{|c|}{ System A } & Neutral & \multicolumn{2}{|c|}{ System B } & \multicolumn{2}{|c|}{ System A } & Neutral & \multicolumn{2}{|c|}{ System B } \\
\hline POINTER(base) & $57.7 \%$ & $19.9 \%$ & $22.4 \%$ & CGMH & PoInter(base) & $54.2 \%$ & $20.0 \%$ & $25.8 \%$ & CGMH \\
\hline PoInter(base) & $52.7 \%$ & $24.1 \%$ & $23.2 \%$ & NMSTG & Pointer(base) & $59.0 \%$ & $22.8 \%$ & $18.2 \%$ & NMSTG \\
\hline POINTER(base) & $16.6 \%$ & $20.0 \%$ & $63.4 \%$ & Human & PoInter(base) & $24.0 \%$ & $26.1 \%$ & $49.9 \%$ & Human \\
\hline \multicolumn{10}{|c|}{ Informativeness: $A$ and $B$, which is more informative? } \\
\hline \multicolumn{5}{|c|}{ News dataset } & \multicolumn{5}{|c|}{ Yelp dataset } \\
\hline \multicolumn{2}{|c|}{ System A } & Neutral & \multicolumn{2}{|c|}{ System B } & \multicolumn{2}{|c|}{ System A } & Neutral & \multicolumn{2}{|c|}{ System B } \\
\hline POINTER(base) & $70.4 \%$ & $12.8 \%$ & $16.8 \%$ & CGMH & POINTER(base) & $69.9 \%$ & $10.9 \%$ & $19.3 \%$ & CGMH \\
\hline POINTER(base) & $\mathbf{5 7 . 7 \%}$ & $18.7 \%$ & $23.6 \%$ & NMSTG & POINTER(base) & $65.2 \%$ & $18.1 \%$ & $16.7 \%$ & NMSTG \\
\hline PoInter(base) & $31.7 \%$ & $19.0 \%$ & $49.4 \%$ & Human & POINTER(base) & $32.8 \%$ & $19.0 \%$ & $48.2 \%$ & Human \\
\hline
\end{tabular}

Table 5: Human Evaluation on two datasets for semantic consistency, fluency and informativeness, showing preferences (\%) for our POINTER(base) model vis-à-vis baselines and real human responses. Numbers in bold indicate the most preferred systems. Differences in mean preferences are statistically significant at $p \leq 0.00001$.

on the Yelp dataset, where the goal is to generate a long-form text from more constraints. Generating a longer piece of text with more lexical constraints is generally more challenging, since the model needs to capture the long-term dependency structure from the text, and effectively conjure up with a plan to realize the generation. Results of automatic evaluation are provided in Table 2 (lower). Generated examples are shown in Table 4 and Appendix C. Generally, the generation from our model effectively considers all the lexical constraints, and is semantically more coherent and grammatically more fluent, compared with the baseline methods. The automatic evaluation results is generally consistent with the observations from News dataset, with an exception that Dist-n scores is much lower than the human Dist-n scores. Compared with greedy approach, at a cost of efficiency, ILBS is typically more concise and contains less repeated information, a defect the greedy approach occasionally suffers (e.g., Table 4, "delicious and delicious").

For both datasets, most of the generations converges with in 4 stages. We perform additional experiments on zero-shot generation from the pretrained model on both datasets, to test the versatility of pre-training. The generated sentences, albeit Wiki-like, are relatively fluent and coherent (see examples in Appendix B and C), and yield relatively high relevance scores (see Appendix E for details). Interestingly, less informative constraints are able to be expanded to coherent sentences. Given the constraint is to from, our model generates "it is oriented to its east, but from the west".

The autoregressive soft-constraint baseline(Gao et al., 2020) has no guarantee that it will cover all keywords in the given order, thus we omit it in the Table 2. For this baseline, the percentage of keywords that appear in the outputs are $57 \%$ and $43 \%$ for News and Yelp datasets, respectively. With the similar model size (117M), this baselines performance is worse than ours approach in automatic metrics for News dataset (BLEU4: $2.99 \rightarrow 1.74$; NIST4: $3.22 \rightarrow 1.10$; METEOR: $16 \% \rightarrow 9 \%$; DIST2: $61 \% \rightarrow 58 \%$; PPL: $66 \rightarrow 84$ ). The performance gap in Yelp dataset is even larger due to more lexical constraints.

Human Evaluation Using a public crowdsourcing platform (UHRS), we conducted a human evaluation of 400 randomly sampled outputs (out of $1 \mathrm{k}$ test set) of CGMH, NMSTG and our base and large models with greedy decoding. Systems were paired and each pair of system outputs was randomly presented (in random order) to 5 crowdsourced judges, who ranked the outputs pairwise for coherence, informativeness and fluency using 


\begin{tabular}{l|c|c}
\hline Model & Training & Inference \\
\hline CGMH & 4382 toks/s & $33 \mathrm{~h}$ \\
NMSTG & 357 toks/s & $487 \mathrm{~s}$ \\
POINTER & 5096 toks/s & $94 \mathrm{~s}$ \\
\hline
\end{tabular}

Table 6: Speed comparison. "toks/s" represents tokens per second. Inference time is computed on 1000 test examples. POINTER uses (greedy, base)

a 5-point Likert-like scale. The human evaluation template is provided in Appendix G. The overall judge preferences for fluency, informativeness and semantic coherence are presented as percentages of the total "vote" in Table 5. P-values are all $p_{i} 0.00001$ (line 721), computed using 10000 bootstrap replications. For inter-annotator agreement, Krippendorff's alpha is 0.23 on the News dataset and 0.18 on the Yelp dataset. Despite the noise, the judgments show a strong across-the-board preference for POINTER(base) over the two baseline systems on all categories. A clear preference for the human ground truth over our method is also observed. The base and large models show comparable human judge preferences on the News dataset, while human judges clearly prefer the large model on Yelp data (see Appendix D for more details).

Running-time Comparison One of the motivations of this work is that at each stage the generation can be parallel, leading to a significant reduction in training and inference. We compare the model training time and the inference decoding time of all the methods on the Yelp dataset, and summarize the results in Table 6 . The evaluation is based on a single Nvidia V100 GPU. Training time for CGMH and POINTER is relatively fast, while NMSTG processes fewer tokens per second since it needs to generate a tree-like structure for each sentence. With respect to inference time, CGMH is slow, as it typically needs hundreds of sampling iterations to decode one sentence.

We note there is no theoretical guarantee of $\mathcal{O}(\log N)$ time complexity for our method. However, our approach encourages filling as many slots as possible at each stage, which permits enables the model to achieve an empirical $\mathcal{O}(\log N)$ speed. In our experiment $98 \%$ of generations end within 4 stages.

Note that our method in Table 6 uses greedy decoding. ILBS is around 20 times slower than greedy. The large model is around 3 times slower than the base model.

\section{Conclusion}

We have presented POINTER, a simple yet powerful approach to generating text from a given set of lexical constraints in a non-autoregressive manner. The proposed method leverages a large-scale pre-trained model (such as BERT initialization and our insertion-based pre-training on Wikipedia) to generate text in a progressive manner using an insertion-based Transformer. Both automatic and human evaluation demonstrate the effectiveness of POINTER. In future work, we hope to leverage sentence structure, such as the use of constituency parsing, to further enhance the design of the progressive hierarchy. Our model can be also extended to allow inflected/variant forms and arbitrary ordering of given lexical constraints.

\section{References}

Daniel Adiwardana, Minh-Thang Luong, David R So, Jamie Hall, Noah Fiedel, Romal Thoppilan, Zi Yang, Apoorv Kulshreshtha, Gaurav Nemade, Yifeng Lu, et al. 2020. Towards a human-like open-domain chatbot. arXiv preprint arXiv:2001.09977.

Dzmitry Bahdanau, Kyunghyun Cho, and Yoshua Bengio. 2015. Neural machine translation by jointly learning to align and translate. In ICLR.

Richard Bellman. 1954. The theory of dynamic programming. Technical report, Rand corp santa monica ca.

Jon Bentley. 1984. Programming pearls: algorithm design techniques. Communications of the ACM, 27(9):865-873.

Ricardo Campos, Vítor Mangaravite, Arian Pasquali, Alípio Jorge, Célia Nunes, and Adam Jatowt. 2020. Yake! keyword extraction from single documents using multiple local features. Information Sciences.

Ricardo Campos, Vítor Mangaravite, Arian Pasquali, Alípio Mário Jorge, Célia Nunes, and Adam Jatowt. 2018. Yake! collection-independent automatic keyword extractor. In European Conference on Information Retrieval.

William Chan, Nikita Kitaev, Kelvin Guu, Mitchell Stern, and Jakob Uszkoreit. 2019. Kermit: Generative insertion-based modeling for sequences. arXiv preprint arXiv:1906.01604.

Liqun Chen, Yizhe Zhang, Ruiyi Zhang, Chenyang Tao, Zhe Gan, Haichao Zhang, Bai Li, Dinghan Shen, Changyou Chen, and Lawrence Carin. 2019a. Improving sequence-to-sequence learning via optimal transport. In ICLR. 
Yen-Chun Chen, Zhe Gan, Yu Cheng, Jingzhou Liu, and Jingjing Liu. 2019b. Distilling the knowledge of bert for text generation. arXiv preprint arXiv:1911.03829.

Woon Sang Cho, Pengchuan Zhang, Yizhe Zhang, Xiujun Li, Michel Galley, Chris Brockett, Mengdi Wang, and Jianfeng Gao. 2018. Towards coherent and cohesive long-form text generation. arXiv preprint arXiv:1811.00511.

Kevin Clark, Minh-Thang Luong, Quoc V. Le, and Christopher D. Manning. 2020. ELECTRA: Pretraining text encoders as discriminators rather than generators. In ICLR.

Jacob Devlin, Ming-Wei Chang, Kenton Lee, and Kristina Toutanova. 2019. Bert: Pre-training of deep bidirectional transformers for language understanding. In $N A A C L$.

George Doddington. 2002. Automatic evaluation of machine translation quality using n-gram cooccurrence statistics. In Proceedings of the second international conference on Human Language Technology Research.

Chris Donahue, Mina Lee, and Percy Liang. 2020. Enabling language models to fill in the blanks. arXiv preprint arXiv:2005.05339.

Li Dong, Nan Yang, Wenhui Wang, Furu Wei, Xiaodong Liu, Yu Wang, Jianfeng Gao, Ming Zhou, and Hsiao-Wuen Hon. 2019. Unified language model pre-training for natural language understanding and generation. In NeurIPS.

Angela Fan, Mike Lewis, and Yann Dauphin. 2018. Hierarchical neural story generation. In $A C L$.

Xiang Gao, Michel Galley, and Bill Dolan. 2020. Mixingboard: a knowledgeable stylized integrated text generation platform. In ACL, system demonstration.

Marjan Ghazvininejad, Omer Levy, Yinhan Liu, and Luke Zettlemoyer. 2019. Mask-predict: Parallel decoding of conditional masked language models. In EMNLP.

David Gries. 1982. A note on a standard strategy for developing loop invariants and loops. Science of Computer Programming.

Jiatao Gu, James Bradbury, Caiming Xiong, Victor OK Li, and Richard Socher. 2018. Non-autoregressive neural machine translation. In ICLR.

Jiatao Gu, Qi Liu, and Kyunghyun Cho. 2019. Insertion-based decoding with automatically inferred generation order. TACL.

Jiatao $\mathrm{Gu}$, Zhengdong $\mathrm{Lu}$, Hang $\mathrm{Li}$, and Victor $\mathrm{OK}$ Li. 2016. Incorporating copying mechanism in sequence-to-sequence learning. arXiv preprint arXiv:1603.06393.
Chris Hokamp and Qun Liu. 2017. Lexically constrained decoding for sequence generation using grid beam search. In $A C L$.

J Edward Hu, Huda Khayrallah, Ryan Culkin, Patrick Xia, Tongfei Chen, Matt Post, and Benjamin Van Durme. 2019. Improved lexically constrained decoding for translation and monolingual rewriting. In NAACL.

Katharina Kann, Sascha Rothe, and Katja Filippova. 2018. Sentence-level fluency evaluation: References help, but can be spared! arXiv preprint arXiv:1809.08731.

Jungo Kasai, James Cross, Marjan Ghazvininejad, and Jiatao Gu. 2020. Parallel machine translation with disentangled context transformer. arXiv preprint arXiv:2001.05136.

Nitish Shirish Keskar, Bryan McCann, Lav Varshney, Caiming Xiong, and Richard Socher. 2019. CTRL - A Conditional Transformer Language Model for Controllable Generation. arXiv preprint arXiv:1909.05858.

D. Kingma and J. Ba. 2015. Adam: A method for stochastic optimization. In ICLR.

Alon Lavie and Abhaya Agarwal. 2007. Meteor: An automatic metric for mt evaluation with high levels of correlation with human judgments. In Proceedings of the Second Workshop on Statistical Machine Translation.

Jason Lee, Elman Mansimov, and Kyunghyun Cho. 2018. Deterministic non-autoregressive neural sequence modeling by iterative refinement. EMNLP.

Mike Lewis, Yinhan Liu, Naman Goyal, Marjan Ghazvininejad, Abdelrahman Mohamed, Omer Levy, Ves Stoyanov, and Luke Zettlemoyer. 2019. Bart: Denoising sequence-to-sequence pre-training for natural language generation, translation, and comprehension. arXiv preprint arXiv:1910.13461.

Dianqi Li, Yizhe Zhang, Hao Peng, Liqun Chen, Chris Brockett, Ming-Ting Sun, and Bill Dolan. 2020. Contextualized perturbation for textual adversarial attack. arXiv preprint arXiv:2009.07502.

Jiwei Li, Michel Galley, Chris Brockett, Jianfeng Gao, and Bill Dolan. 2016. A diversity-promoting objective function for neural conversation models. In $N A A C L$

Yinhan Liu, Myle Ott, Naman Goyal, Jingfei Du, Mandar Joshi, Danqi Chen, Omer Levy, Mike Lewis, Luke Zettlemoyer, and Veselin Stoyanov. 2019. Roberta: A robustly optimized bert pretraining approach. arXiv preprint arXiv:1907.11692.

Xuezhe Ma, Chunting Zhou, Xian Li, Graham Neubig, and Eduard Hovy. 2019. Flowseq: Nonautoregressive conditional sequence generation with generative flow. arXiv preprint arXiv:1909.02480. 
Elman Mansimov, Alex Wang, and Kyunghyun Cho. 2019. A generalized framework of sequence generation with application to undirected sequence models. arXiv preprint arXiv:1905.12790.

Ning Miao, Hao Zhou, Lili Mou, Rui Yan, and Lei Li 2019. Cgmh: Constrained sentence generation by metropolis-hastings sampling. In $A A A I$.

Lili Mou, Yiping Song, Rui Yan, Ge Li, Lu Zhang, and Zhi Jin. 2016. Sequence to backward and forward sequences: A content-introducing approach to generative short-text conversation. arXiv preprint arXiv:1607.00970.

Kishore Papineni, Salim Roukos, Todd Ward, and WeiJing Zhu. 2002. Bleu: a method for automatic evaluation of machine translation. In $A C L$.

Matt Post and David Vilar. 2018. Fast lexically constrained decoding with dynamic beam allocation for neural machine translation. In $N A A C L$.

Lianhui Qin, Michel Galley, Chris Brockett, Xiaodong Liu, Xiang Gao, Bill Dolan, Yejin Choi, and Jianfeng Gao. 2019. Conversing by reading: Contentful neural conversation with on-demand machine reading. In $A C L$.

A. Radford, J. Wu, R. Child, D. Luan, D. Amodei, and I. Sutskever. 2018. Language models are unsupervised multitask learners. Technical report, OpenAI.

Colin Raffel, Noam Shazeer, Adam Roberts, Katherine Lee, Sharan Narang, Michael Matena, Yanqi Zhou, Wei Li, and Peter J. Liu. 2019. Exploring the limits of transfer learning with a unified text-to-text transformer. arXiv preprint arXiv:1910.10683.

Arthur Richards and Jonathan P How. 2002. Aircraft trajectory planning with collision avoidance using mixed integer linear programming. In Proceedings of American Control Conference.

Kaitao Song, Xu Tan, Tao Qin, Jianfeng Lu, and TieYan Liu. 2019. Mass: Masked sequence to sequence pre-training for language generation. arXiv preprint arXiv:1905.02450.

Mitchell Stern, William Chan, Jamie Kiros, and Jakob Uszkoreit. 2019. Insertion transformer: Flexible sequence generation via insertion operations. arXiv preprint arXiv:1902.03249.

Zhiqing Sun, Zhuohan Li, Haoqing Wang, Di He, Zi Lin, and Zhihong Deng. 2019. Fast structured decoding for sequence models. In NeurIPS.

Jianheng Tang, Tiancheng Zhao, Chenyan Xiong, Xiaodan Liang, Eric P. Xing, and Zhiting Hu. 2019. Target-guided open-domain conversation. In $A C L$.

Sean Welleck, Kianté Brantley, Hal Daumé III, and Kyunghyun Cho. 2019. Non-monotonic sequential text generation. arXiv preprint arXiv:1902.02192.
Felix Wu, Angela Fan, Alexei Baevski, Yann N Dauphin, and Michael Auli. 2019. Pay less attention with lightweight and dynamic convolutions. arXiv preprint arXiv:1901.10430.

Yonghui Wu, Mike Schuster, Zhifeng Chen, Quoc V Le, Mohammad Norouzi, Wolfgang Macherey, Maxim Krikun, Yuan Cao, Qin Gao, Klaus Macherey, et al. 2016. Google's neural machine translation system: Bridging the gap between human and machine translation. arXiv preprint arXiv:1609.08144.

Zhilin Yang, Zihang Dai, Yiming Yang, Jaime Carbonell, Russ R Salakhutdinov, and Quoc V Le. 2019. Xlnet: Generalized autoregressive pretraining for language understanding. In NeurIPS.

Lili Yao, Nanyun Peng, Ralph Weischedel, Kevin Knight, Dongyan Zhao, and Rui Yan. 2019. Planand-write: Towards better automatic storytelling. In $A A A I$, volume 33 , pages $7378-7385$.

Rowan Zellers, Ari Holtzman, Hannah Rashkin, Yonatan Bisk, Ali Farhadi, Franziska Roesner, and Yejin Choi. 2019. Defending against neural fake news. In NeurIPS.

Yizhe Zhang, Michel Galley, Jianfeng Gao, Zhe Gan, Xiujun Li, Chris Brockett, and Bill Dolan. 2018. Generating informative and diverse conversational responses via adversarial information maximization. In NeurIPS.

Yizhe Zhang, Dinghan Shen, Guoyin Wang, Zhe Gan, Ricardo Henao, and Lawrence Carin. 2017. Deconvolutional paragraph representation learning. In NeurIPS.

Yizhe Zhang, Siqi Sun, Michel Galley, Yen-Chun Chen, Chris Brockett, Xiang Gao, Jianfeng Gao, Jingjing Liu, and Bill Dolan. 2020. Dialogpt: Large-scale generative pre-training for conversational response generation. In ACL (system demonstration). 


\section{Appendix}

\section{A Baseline and Experimental Details}

For NMSTG, we first convert the lexical constraints into a prefix sub-tree, and then sample a sentence to complete the sub-tree. We use the default settings suggested by the authors, and use an LSTM with hidden size of 1024 as the text generator, and select the best performed variants (annealed) as our baseline. For CGMH, we use their default setting, which uses an LSTM with hidden size of 300, and set the vocabulary size as $50 \mathrm{k}$. Both models are trained until the evaluation loss does not decrease. During inference, we run CGMH for 500 iterations with default hyperparameters.

For experiment setup, we employ the tokenizer from BERT, and use WordPiece Embeddings (Wu et al., 2016) with a 30k token vocabulary for all the tasks. A special no-insertion token [NOI] is added to the vocabulary. We utilize the BERT-base and BERT-large models with 12 self-attention layers and 768 hidden dimensions as our model initialization. Each model is trained until there is no progress on the validation loss. We use a learning rate of 3e-5 without any warming-up schedule for all the training procedures. The optimization algorithm is Adam (Kingma and Ba, 2015). We pretrain our model on the Wiki dataset for 2 epochs, and fine-tune on the News and Yelp datasets for around 10 epochs.

\section{B Additional Generated Examples for News Dataset}

We provide two examples on News dataset for how the model progressively generates the sentences in Table 7. All the generations are from the POINTER large model using greedy decoding.

In this section, we also provide some additional examples from the $1 \mathrm{k}$ news test data.

\begin{tabular}{|c|c|}
\hline Stage & Generated text sequence \\
\hline $0\left(X^{0}\right)$ & aware negative immediately sites \\
\hline $1\left(X^{1}\right)$ & $\begin{array}{l}\text { if aware posts negative should immediately any } \\
\text { sites posts }\end{array}$ \\
\hline $2\left(X^{2}\right)$ & $\begin{array}{l}\text { would if user aware that posts have negative } \\
\text { impact should immediately related any these } \\
\text { sites remove posts }\end{array}$ \\
\hline $3\left(X^{3}\right)$ & $\begin{array}{l}\text { this would prefer if the user is aware that the } \\
\text { posts have a negative impact and should be im- } \\
\text { mediately related to any of these sites and re- } \\
\text { move those posts. }\end{array}$ \\
\hline Stage & Generated text sequence \\
\hline $0\left(X^{0}\right)$ & estate pay stay policy \\
\hline $1\left(X^{1}\right)$ & $\begin{array}{l}\text { also estate agents pay amount stay same policy } \\
\text { assets }\end{array}$ \\
\hline $2\left(X^{2}\right)$ & $\begin{array}{l}\text { it also required estate agents who pay same } \\
\text { amount cash stay with same policy all assets }\end{array}$ \\
\hline $3\left(X^{3}\right)$ & $\begin{array}{l}\text { but it would also be required for estate agents, } \\
\text { who must pay the same amount of cash but stay } \\
\text { with the same policy for all other assets . }\end{array}$ \\
\hline
\end{tabular}

Table 7: Example of the progressive generation process with multiple stages from the POINTER model. New additions at each stage are marked as blue.

\begin{tabular}{l|l}
\hline Keywords & aware negative immediately sites \\
\hline ORACLE & $\begin{array}{l}\text { where we become aware of any accounts that } \\
\text { may be negative, we immediately contact } \\
\text { companies such as Instagram, although we } \\
\text { have no control over what they allow on their } \\
\text { sites . }\end{array}$ \\
\hline CGMH & $\begin{array}{l}\text { not even aware of negative events including } \\
\text { video events immediately at stations, Face- } \\
\text { book sites. }\end{array}$ \\
\hline NMSTG & $\begin{array}{l}\text { health providers in a country for England are } \\
\text { aware of small health systems - and not non } \\
\text { - health care but all negative is immediately } \\
\text { treated by heads of businesses and depart- } \\
\text { ments in the sites . }\end{array}$ \\
\hline $\begin{array}{l}\text { POINTER } \\
\text { (Greedy, } \\
\text { base) }\end{array}$ & $\begin{array}{l}\text { 'if users are aware of the negative impact } \\
\text { of blocking, how can they so immediately } \\
\text { ban these sites ?' the researchers wrote }\end{array}$ \\
\hline $\begin{array}{l}\text { POINTER } \\
\text { (ILBS, } \\
\text { base) }\end{array}$ & $\begin{array}{l}\text { if the users are aware of or the negative mes- } \\
\text { sages, they can immediately be transferred } \\
\text { to other sites . }\end{array}$ \\
\hline $\begin{array}{l}\text { POINTER } \\
\text { (Greedy, } \\
\text { Large) }\end{array}$ & $\begin{array}{l}\text { this would prefer if the user is aware that the } \\
\text { posts have a negative impact and should be } \\
\text { immediately related to any of these sites and } \\
\text { remove those posts }\end{array}$ \\
\hline $\begin{array}{l}\text { Wiki zero- } \\
\text { shot }\end{array}$ & $\begin{array}{l}\text { he is not aware of the negative, and will } \\
\text { immediately go to the positive sites } .\end{array}$ \\
\hline
\end{tabular}




\begin{tabular}{l|l}
\hline Keywords & children fault left charge \\
\hline ORACLE & $\begin{array}{l}\text { my relationship with my children was se- } \\
\text { riously affected as they were told time and } \\
\text { again that everything was my fault, they } \\
\text { were even left 'in charge' of me if my wife } \\
\text { went out of the house. }\end{array}$ \\
\hline CGMH & $\begin{array}{l}\text { his two children are the rare fault that left } \\
\text { the police charge }\end{array}$ \\
\hline NMSTG & $\begin{array}{l}\text { but despite children from hospitals to last } \\
\text { one by fault backing this month, there have } \\
\text { arrived as Mr Hunt has been left charge . }\end{array}$ \\
\hline $\begin{array}{l}\text { POINTER } \\
\text { (Greedy, } \\
\text { base) }\end{array}$ & $\begin{array}{l}\text { but i found that these children were not at } \\
\text { school however this was not their fault, and } \\
\text { if so they were left without a parent in charge } \\
\text { - }\end{array}$ \\
\hline $\begin{array}{l}\text { POINTER } \\
\text { (ILBS, } \\
\text { base) }\end{array}$ & $\begin{array}{l}\text { but my lovely wife and children consider } \\
\text { that it is not our own fault and we should not } \\
\text { be left alone in charge } .\end{array}$ \\
\hline $\begin{array}{l}\text { POINTER } \\
\text { (Greedy, } \\
\text { Large) }\end{array}$ & $\begin{array}{l}\text { i said to my children : it's not his fault the } \\
\text { parents left him ; the parents should be in } \\
\text { charge of him } .\end{array}$ \\
\hline $\begin{array}{l}\text { Wiki zero- } \\
\text { shot }\end{array}$ & $\begin{array}{l}\text { but for the children who are not at a fault } \\
\text { they are left behind on the charge }\end{array}$ \\
\hline
\end{tabular}

\begin{tabular}{l|l}
\hline Keywords & managers cut costs million \\
\hline ORACLE & $\begin{array}{l}\text { he was the third of four managers sent in } \\
\text { to cut costs and deal with the city s \$ 13 } \\
\text { million deficit . }\end{array}$ \\
\hline CGMH & $\begin{array}{l}\text { the managers, who tried to cut off their } \\
\text { costs, added 20 million euros }\end{array}$ \\
\hline NMSTG & $\begin{array}{l}\text { business managers cut demand for more ex- } \\
\text { pensive costs in 2017 - by October - is around }\end{array}$ \\
$\begin{array}{l}5 \text { million 8 per cent, and has fallen by 0. 3 } \\
\text { per cent in January and 2017 . }\end{array}$ \\
\hline $\begin{array}{l}\text { POINTER } \\
\text { breedy, }\end{array}$ & $\begin{array}{l}\text { under one of its general managers, the firm } \\
\text { had already cut its annual operating costs } \\
\text { from \$ 13.5 million to six million euros . }\end{array}$ \\
\hline $\begin{array}{l}\text { POINTER } \\
\text { (ILBS, } \\
\text { base) }\end{array}$ & $\begin{array}{l}\text { and last month, the managers announced } \\
\text { that it had cut its operating costs by \$ 30 } \\
\text { million . }\end{array}$ \\
\hline $\begin{array}{l}\text { POINTER } \\
\text { (Greedy, }\end{array}$ & $\begin{array}{l}\text { the biggest expense is for the managers } \\
\text { where it plans to cut their annual manage- } \\
\text { ment costs from \$ 18 . 5 million to \$ 12 } \\
\text { million . }\end{array}$ \\
\hline $\begin{array}{l}\text { Wikge) } \\
\text { shot }\end{array}$ & $\begin{array}{l}\text { but then he and all of his managers agreed } \\
\text { to cut off all of the operating costs by about } \\
1 \text { million . }\end{array}$ \\
\hline
\end{tabular}

\begin{tabular}{l|l}
\hline Keywords & estate pay stay policy \\
\hline ORACLE & $\begin{array}{l}\text { how many people on the estate does he think } \\
\text { will be affected by the new pay - to - stay } \\
\text { policy ? }\end{array}$ \\
\hline CGMH & $\begin{array}{l}\text { an economic estate developer that could pay } \\
\text { for it is that a stay policy }\end{array}$ \\
\hline NMSTG & $\begin{array}{l}\text { as estate owners , they cannot pay for house- } \\
\text { holds for hundreds of middle - income prop- } \\
\text { erty, buyers stay in retail policy . }\end{array}$ \\
\hline $\begin{array}{l}\text { POINTER } \\
\text { (Greedy, } \\
\text { base) }\end{array}$ & $\begin{array}{l}\text { if you buy new buildings from real estate } \\
\text { company, you may have to pay down a mort- } \\
\text { gage and stay with the policy for financial } \\
\text { reasons . }\end{array}$ \\
\hline $\begin{array}{l}\text { POINTER } \\
\text { (ILBS, } \\
\text { base) }\end{array}$ & $\begin{array}{l}\text { but no matter what foreign buyers do , real } \\
\text { estate agents will have to pay a small fee to } \\
\text { stay consistent with the policy . }\end{array}$ \\
\hline $\begin{array}{l}\text { POINTER } \\
\text { (Greedy, } \\
\text { Large) }\end{array}$ & $\begin{array}{l}\text { but it would also be required for estate agents } \\
\text { who must pay a larger amount of cash but } \\
\text { stay with the same policy for all other assets } \\
\text {. }\end{array}$ \\
\hline $\begin{array}{l}\text { Wiki zero- } \\
\text { shot }\end{array}$ & $\begin{array}{l}\text { however , his real estate agent agreed to pay } \\
\text { him for the stay under the same policy } .\end{array}$ \\
\hline
\end{tabular}

\begin{tabular}{|c|c|}
\hline Keywords & looked report realized wife \\
\hline ORACLE & $\begin{array}{l}\text { i looked at the report and saw her name, } \\
\text { and that's when I realized it was my ex-wife } \\
\text {. }\end{array}$ \\
\hline CGMH & $\begin{array}{l}\text { he looked at the report and said he realized } \\
\text { that if his wife Jane }\end{array}$ \\
\hline NMSTG & $\begin{array}{l}\text { i looked at my report about before I realized } \\
\text { I return to travel holidays but - it doesn' } t \\
\text { haven' } t \text { made anything like my wife. }\end{array}$ \\
\hline $\begin{array}{l}\text { POINTER } \\
\text { (Greedy, } \\
\text { base) }\end{array}$ & $\begin{array}{l}\text { when } i \text { turned and looked at a file report } \\
\text { from the airport and realized it was not my } \\
\text { wife and daughter. }\end{array}$ \\
\hline $\begin{array}{l}\text { POINTER } \\
\text { (ILBS, } \\
\text { base) }\end{array}$ & $\begin{array}{l}\text { when } i \text { turned around and looked down at the } \\
\text { pictures from the report, i realized that it } \\
\text { was my wife. }\end{array}$ \\
\hline $\begin{array}{l}\text { POINTER } \\
\text { (Greedy, } \\
\text { Large) }\end{array}$ & $\begin{array}{l}\text { however, when they looked at the details of } \\
\text { the report about this murder, they quickly } \\
\text { realized that the suspect was not with his } \\
\text { wife or his partner. }\end{array}$ \\
\hline $\begin{array}{l}\text { Wiki zero- } \\
\text { shot }\end{array}$ & $\begin{array}{l}\text { but when he looked up at the report, he } \\
\text { realized that it was not his wife. }\end{array}$ \\
\hline
\end{tabular}




\begin{tabular}{l|l}
\hline Keywords & time claim tax year \\
\hline ORACLE & $\begin{array}{l}\text { walker says there is still time to claim this } \\
\text { higher protection if you haven 't already as } \\
\text { the deadline is the end of the 2016 / } 2017 \text { tax } \\
\text { year . }\end{array}$ \\
\hline CGMH & $\begin{array}{l}\text { " two states, one - time voters can claim a } \\
\text { federal tax year }\end{array}$ \\
\hline NMSTG & $\begin{array}{l}\text { this time they had three to claim of an equal } \\
\text { tax and } 34 \text { women at which indicated they } \\
\text { should leave that over the year of } 16 .\end{array}$ \\
\hline $\begin{array}{l}\text { POINTER } \\
\text { (Greedy, } \\
\text { base) }\end{array}$ & $\begin{array}{l}\text { it is the very first time in history that trump } \\
\text { will ever claim over } \$ 400 \text { million in federal } \\
\text { income tax that he had held last year, the } \\
\text { same report says . }\end{array}$ \\
\hline $\begin{array}{l}\text { POINTER } \\
\text { (ILBS, } \\
\text { base) }\end{array}$ & $\begin{array}{l}\text { is this the very first time someone has to } \\
\text { claim federal income tax twice in a single } \\
\text { year ? }\end{array}$ \\
\hline $\begin{array}{l}\text { POINTER } \\
\text { (Greedy, }\end{array}$ & $\begin{array}{l}\text { this is not for the first time that the scottish } \\
\text { government was able to claim tax cuts of } \\
\text { thousands of pounds a year to pay . }\end{array}$ \\
\hline $\begin{array}{l}\text { Wiki zero- } \\
\text { shot }\end{array}$ & $\begin{array}{l}\text { but at the time the claim was that the same } \\
\text { sales tax that was from the previous fiscal } \\
\text { year } .\end{array}$ \\
\hline
\end{tabular}

\begin{tabular}{l|l}
\hline Keywords & made year resolution managed \\
\hline ORACLE & $\begin{array}{l}\text { i once made this my new year' s resolution } \\
\text { and it is the only one that I' ve actually ever } \\
\text { managed to keep . }\end{array}$ \\
\hline CGMH & $\begin{array}{l}\text { indeed, as he made up the previous year } \\
\text { the GOP resolution was managed }\end{array}$ \\
\hline NMSTG & $\begin{array}{l}\text { while additional sanctions had been issued } \\
\text { last week made a year from the latest reso- } \\
\text { lution, Russia 's Russian ministers have but } \\
\text { have managed } .\end{array}$ \\
\hline $\begin{array}{l}\text { POINTER } \\
\text { (Greedy, } \\
\text { base) }\end{array}$ & $\begin{array}{l}\text { no progress has been made in syria since } \\
\text { the security council started a year ago } \\
\text { when a resolution expressed confidence that } \\
\text { moscow managed to save aleppo . }\end{array}$ \\
\hline $\begin{array}{l}\text { POINTER } \\
\text { (ILBS, } \\
\text { base) }\end{array}$ & $\begin{array}{l}\text { and the enormous progress we have made } \\
\text { over the last year is to bring about a resolu- } \\
\text { tion that has not been managed . }\end{array}$ \\
\hline $\begin{array}{l}\text { POINTER } \\
\text { (Greedy, }\end{array}$ & $\begin{array}{l}\text { the obama administration, which made a } \\
\text { similar call earlier this year and has also } \\
\text { voted against a resolution to crack down on } \\
\text { the funding, managed to recover it . }\end{array}$ \\
\hline $\begin{array}{l}\text { Wiki zero- } \\
\text { shot }\end{array}$ & $\begin{array}{l}\text { but despite all the same changes made both } \\
\text { in both the previous fiscal year, and by the } \\
\text { un resolution itself, only the federal govern- } \\
\text { ment managed ... }\end{array}$ \\
\hline
\end{tabular}

\begin{tabular}{l|l}
\hline Keywords & model years big drama \\
\hline ORACLE & $\begin{array}{l}\text { the former model said : " I haven ' t seen } \\
\text { him in so many years, I can ' t make a big } \\
\text { drama out of it." }\end{array}$ \\
\hline CGMH & $\begin{array}{l}\text { the " model " continues, like many years of } \\
\text { sexual and big drama going }\end{array}$ \\
\hline NMSTG & $\begin{array}{l}\text { after model two years and did it like, could } \\
\text { we already get bigger than others in a big } \\
\text { drama ? }\end{array}$ \\
\hline $\begin{array}{l}\text { POINTER } \\
\text { (Greedy, } \\
\text { base) }\end{array}$ & $\begin{array}{l}\text { but i am a good role model, who has been } \\
\text { around for 10 years now , and that is a big } \\
\text { example of what i can do in drama on screen } \\
\text {. }\end{array}$ \\
\hline $\begin{array}{l}\text { POINTER } \\
\text { (ILBS, } \\
\text { base) }\end{array}$ & $\begin{array}{l}\text { but the young actress and model, for } 15 \\
\text { years, made a very big impact on the drama } \\
\text {. }\end{array}$ \\
\hline $\begin{array}{l}\text { POINTER } \\
\text { (Greedy, } \\
\text { Large) }\end{array}$ & $\begin{array}{l}\text { i have seen the different model she recom- } \\
\text { mends of over years, but it's no big change } \\
\text { in the drama after all . }\end{array}$ \\
\hline $\begin{array}{l}\text { Wiki zero- } \\
\text { shot }\end{array}$ & $\begin{array}{l}\text { she was a model actress for many years and } \\
\text { was a big star in the drama . }\end{array}$ \\
\hline
\end{tabular}

\begin{tabular}{|c|c|}
\hline Keywords & club believed centre window \\
\hline ORACLE & $\begin{array}{l}\text { the club are believed to be keen on bringing } \\
\text { in cover at centre - back during the current } \\
\text { transfer window, with a loan move most } \\
\text { likely. }\end{array}$ \\
\hline CGMH & $\begin{array}{l}\text { the club has also been believed that more } \\
\text { than a new centre - up window }\end{array}$ \\
\hline NMSTG & $\begin{array}{l}\text { one club believed it was not clear that the } \\
\text { centre would hold place on the window until } \\
\text { there were no cases that they had heard or had } \\
\text { the decision disappeared. }\end{array}$ \\
\hline $\begin{array}{l}\text { POINTER } \\
\text { (Greedy, } \\
\text { base) }\end{array}$ & $\begin{array}{l}\text { he had been talking to the club since he is } \\
\text { believed to have reached the centre spot in } \\
\text { the queue before the january transfer window } \\
\text { was suspended. }\end{array}$ \\
\hline $\begin{array}{l}\text { POINTER } \\
\text { (ILBS, } \\
\text { base) }\end{array}$ & $\begin{array}{l}\text { when he left his old club, chelsea, he was } \\
\text { believed to be at the centre of the transfer } \\
\text { window. }\end{array}$ \\
\hline $\begin{array}{l}\text { POINTER } \\
\text { (Greedy, } \\
\text { Large) }\end{array}$ & $\begin{array}{l}\text { the striker has remained at the club at the } \\
\text { weekend and is increasingly believed to be } \\
\text { available as a centre of the club during the } \\
\text { summer transfer window until january } 2016 \\
\text {. }\end{array}$ \\
\hline $\begin{array}{l}\text { Wiki zero- } \\
\text { shot }\end{array}$ & $\begin{array}{l}\text { during his first club as manager he was } \\
\text { widely believed to be at the centre forward } \\
\text { in the january transfer window. }\end{array}$ \\
\hline
\end{tabular}




\begin{tabular}{l|l}
\hline Keywords & great past decade city \\
\hline ORACLE & $\begin{array}{l}\text { it's been a great time, the past decade or } \\
\text { so, to be the mayor of a major capital city }\end{array}$ \\
\hline CGMH & $\begin{array}{l}\text { the great past decade is that so much of a new } \\
\text { home city }\end{array}$ \\
\hline NMSTG & $\begin{array}{l}\text { i like to thank you for me and I' ve wanted it } \\
\text { to grow in every great past decade over the } \\
\text { city, a very amazing time. }\end{array}$ \\
\hline $\begin{array}{l}\text { POINTER } \\
\text { (Greedy, } \\
\text { base) }\end{array}$ & $\begin{array}{l}\text { this is one of the great cities that he have } \\
\text { visited in the past two decade, the kansas } \\
\text { city, missouri, he says . }\end{array}$ \\
\hline $\begin{array}{l}\text { POINTER } \\
\text { (ILBS, } \\
\text { base) }\end{array}$ & $\begin{array}{l}\text { you don' t feel as great as you' ve been in } \\
\text { the past decade in a major city . }\end{array}$ \\
\hline $\begin{array}{l}\text { POINTER } \\
\text { (Greedy, } \\
\text { Large) }\end{array}$ & $\begin{array}{l}\text { there has been a lot of great work here in the } \\
\text { past few years within more than a decade } \\
\text { done for the city, he says . }\end{array}$ \\
\hline $\begin{array}{l}\text { Wiki zero- } \\
\text { shot }\end{array}$ & $\begin{array}{l}\text { there was a great success in the past during } \\
\text { the last decade for the city . }\end{array}$ \\
\hline
\end{tabular}

\section{Additional Generated Examples for Yelp Dataset}

We provide two examples on Yelp dataset for how the model progressively generates the sentences in Table 8. All the generations are from the POINTER large model using greedy decoding.

We also provide some additional examples from the Yelp test set. The results includes keywords, human oracle, CGMH, NMSTG and our models. For our models, we include POINTER base and large models with greedy decoding and base model with ILBS. The large model with ILBS is time consuming so we omit them from the comparison.

\begin{tabular}{|c|c|}
\hline Stage & Generated text sequence \\
\hline $0\left(X^{0}\right)$ & delicious love mole rice back \\
\hline $1\left(X^{1}\right)$ & $\begin{array}{l}\text { restaurant delicious authentic love dish mole } \\
\text { beans rice definitely back ! }\end{array}$ \\
\hline $2\left(X^{2}\right)$ & $\begin{array}{l}\text { new restaurant so delicious fresh authentic. love } \\
\text { mexican dish called mole with beans and rice } \\
\text { we definitely coming back more! }\end{array}$ \\
\hline $3\left(X^{3}\right)$ & $\begin{array}{l}\text { this new restaurant is so delicious, fresh and } \\
\text { authentic tasting . i love the mexican style dish } \\
\text {, called the mole, with black beans, and white } \\
\text { rice. we will definitely be coming back for more } \\
\text { ! }\end{array}$ \\
\hline Stage & Generated text sequence \\
\hline $0\left(X^{0}\right)$ & joint great food great drinks greater staff \\
\hline $1\left(X^{1}\right)$ & $\begin{array}{l}\text { new joint around great location food variety } \\
\text { great craft drinks unless greater friendly staff ! }\end{array}$ \\
\hline $2\left(X^{2}\right)$ & $\begin{array}{l}\text { is new breakfast joint be around area great, } \\
\text { location excellent food nice variety selections } \\
\text { great of craft, drinks quite unless ask greater . } \\
\text { friendly and staff love! }\end{array}$ \\
\hline $3\left(X^{3}\right)$ & $\begin{array}{l}\text { this is the new modern breakfast joint to be } \\
\text { found around the area . great atmosphere, cen- } \\
\text { tral location and excellent food . nice variety of } \\
\text { selections . great selection of local craft beers } \\
\text {, good drinks . quite cheap unless you ask for } \\
\text { greater price . very friendly patio and fun staff. } \\
\text { love it! }\end{array}$ \\
\hline
\end{tabular}

Table 8: Example of the progressive generation process with multiple stages from the POINTER model. New additions at each stage are marked as blue. 


\begin{tabular}{|c|c|}
\hline Keywords & $\begin{array}{l}\text { service perfect delicious service awe- } \\
\text { some good place }\end{array}$ \\
\hline ORACLE & $\begin{array}{l}\text { yummy excellent service . ordered } \\
\text { the carne asada medium rare. it was } \\
\text { perfect . and delicious . their cus- } \\
\text { tomer service was awesome . they } \\
\text { were so friendly and made sure all } \\
\text { was good. i definitely recommend } \\
\text { this place. }\end{array}$ \\
\hline CGMH & $\begin{array}{l}\text { great service perfect food and deli- } \\
\text { cious service . awesome place and } \\
\text { good place!. }\end{array}$ \\
\hline NMSTG & $\begin{array}{l}\text { service was perfect, delicious and } \\
\text { great service awesome service good } \\
\text { food. this place will go back. }\end{array}$ \\
\hline $\begin{array}{l}\text { POINTER } \\
\text { (Greedy, } \\
\text { base) }\end{array}$ & $\begin{array}{l}\text { excellent food, great service, really } \\
\text { nice atmosphere, perfect amount of } \\
\text { spring rolls, delicious especially the } \\
\text { chicken and eel . the service was } \\
\text { very friendly and the prices are awe- } \\
\text { some too . for a female who loves } \\
\text { good japanese restaurant, this is def- } \\
\text { initely your place! }\end{array}$ \\
\hline $\begin{array}{l}\text { POINTER } \\
\text { (ILBS, } \\
\text { base) }\end{array}$ & $\begin{array}{l}\text { from the food to service . the foods } \\
\text { are perfect, they were delicious } \\
\text { and service is beyond expectation . } \\
\text { christina was awesome, so many } \\
\text { good things about this place. }\end{array}$ \\
\hline $\begin{array}{l}\text { POINTER } \\
\text { (Greedy, } \\
\text { Large) }\end{array}$ & $\begin{array}{l}\text { absolutely loved the food and very } \\
\text { friendly service. i had the chicken } \\
\text {, it was cooked perfect and the } \\
\text { seafood pasta was thick and deli- } \\
\text { cious and not too heavy though. our } \\
\text { service guy at the front bar was so } \\
\text { awesome, he made sure we had a } \\
\text { good time. would definitely recom- } \\
\text { mend to try this place to anyone! }\end{array}$ \\
\hline $\begin{array}{l}\text { Wiki } \\
\text { zero- } \\
\text { shot }\end{array}$ & $\begin{array}{l}\text { he said the service was perfect, } \\
\text { and delicious, and the service that } \\
\text { is awesome, and very good in its } \\
\text { place. }\end{array}$ \\
\hline
\end{tabular}

\begin{tabular}{|c|c|}
\hline Keyword\$ & $\begin{array}{l}\text { good drinks love clients tighter great } \\
\text { service }\end{array}$ \\
\hline ORACLE & $\begin{array}{l}\text { great atmosphere, good food and } \\
\text { drinks . i love coming here in the fall } \\
\text { to spring to meet with clients . their } \\
\text { inside is a little small and makes sum- } \\
\text { mer a bit tighter, but still a great } \\
\text { staff with excellent service. }\end{array}$ \\
\hline CGMH & $\begin{array}{l}\text { good drinks . i love how out clients } \\
\text { are tighter . great customer service }\end{array}$ \\
\hline NMSTG & $\begin{array}{l}\text { such good place with i love the mush- } \\
\text { room drinks . the menu they love the } \\
\text { clients . and tighter out the menu } \\
\text { are great service. }\end{array}$ \\
\hline $\begin{array}{l}\text { POINTER } \\
\text { (Greedy, } \\
\text { base) }\end{array}$ & $\begin{array}{l}\text { this place is good . they have a wide } \\
\text { variety of drinks . this really fits } \\
\text { your taste. love the cozy bar that } \\
\text { allows clients to be able to fit very } \\
\text { tightly and tighter, better blending } \\
\text { with the crowd . great coffee, rea- } \\
\text { sonable prices, and friendly service } \\
\text { ! }\end{array}$ \\
\hline $\begin{array}{l}\text { POINTER } \\
\text { (ILBS, } \\
\text { base) }\end{array}$ & $\begin{array}{l}\text { nice place, with good vibe . nice mix } \\
\text { of drinks and intimate space. what } \\
\text { i really love about was there were so } \\
\text { more mature clients, and they can fit } \\
\text { in a tighter timeline. overall, great } \\
\text { atmosphere and excellent service . }\end{array}$ \\
\hline $\begin{array}{l}\text { POINTER } \\
\text { (Greedy, } \\
\text { Large) }\end{array}$ & $\begin{array}{l}\text { really like this place . has a good dj } \\
\text { good atmosphere and cool drinks } \\
\text { and quite nice lounge area . i love } \\
\text { this idea of having fun on your } \\
\text { clients and rubbing your feet to stand } \\
\text { up tighter than other ones . great va- } \\
\text { riety of drinks and pretty quick ser- } \\
\text { vice at the bar! }\end{array}$ \\
\hline $\begin{array}{l}\text { Wiki } \\
\text { zero- } \\
\text { shot }\end{array}$ & $\begin{array}{l}\text { she is a good at drinks, and in love } \\
\text { for him and all his clients, and he } \\
\text { enjoys a tighter schedule and has a } \\
\text { great food and a generous service. }\end{array}$ \\
\hline
\end{tabular}




\begin{tabular}{l|l}
\hline Keywords & $\begin{array}{l}\text { joint great food great drinks greater } \\
\text { staff }\end{array}$ \\
\hline ORACLE & $\begin{array}{l}\text { apteka is seriously all around the best } \\
\text { vegan joint in the burgh . great food } \\
\text { great drinks, greater staff } .\end{array}$ \\
\hline CGMH & $\begin{array}{l}\text { very cool joint with great food } \\
\text { great drinks and even greater staff } \\
\text { ! }\end{array}$ \\
\hline NMSTG & $\begin{array}{l}\text { awesome joint } \\
\text { great food great drinks . good to } \\
\text { greater and great staff ! }\end{array}$ \\
\hline $\begin{array}{l}\text { POINTER } \\
\text { (Greedy, } \\
\text { base) }\end{array}$ & $\begin{array}{l}\text { my favorite local joint around old } \\
\text { town } \text { great atmosphere, amazing } \\
\text { food, delicious and delicious coffee } \\
\text { great wine selection and delicious } \\
\text { cold drinks , oh and maybe even } \\
\text { a greater patio space and energetic } \\
\text { front desk staff . }\end{array}$ \\
\hline $\begin{array}{l}\text { Zero- } \\
\text { shot }\end{array}$ & $\begin{array}{l}\text { it is a joint owner of the great society } \\
\text { of irish food , and the great britain } \\
\text { and soft drinks , and the greater } \\
\text { britain and its staff . }\end{array}$ \\
\hline $\begin{array}{l}\text { POINTER } \\
\text { (ILBS, } \\
\text { base) }\end{array}$ & $\begin{array}{l}\text { the best breakfast joint in charlotte } \\
\text { great service and amazing food } \\
\text { they have great selection of drinks } \\
\text { that suits the greater aesthetic of the } \\
\text { staff . }\end{array}$ \\
\hline Large) & $\begin{array}{l}\text { this is the new modern breakfast } \\
\text { joint to be found around the area } \\
\text { great atmosphere , central loca- } \\
\text { tion and excellent food . nice vari- } \\
\text { ety of selections . great selection } \\
\text { of local craft beers , good drinks } \\
\text { quite cheap unless you ask for } \\
\text { greater price . very friendly patio } \\
\text { and fun staff . love it ! }\end{array}$ \\
\hline
\end{tabular}

\begin{tabular}{|c|c|}
\hline Keyword\$ & $\begin{array}{l}\text { service polite professional affordable } \\
\text { work safe tree }\end{array}$ \\
\hline ORACLE & $\begin{array}{l}\text { aron's tree service were very polite } \\
\text { and professional . they are very af- } \\
\text { fordable . they arrived a little early } \\
\text { and got right to work . they were } \\
\text { quick and safe . they cleaned up } \\
\text { and hauled out the tree trimmings } \\
\text {. i highly recommend them. }\end{array}$ \\
\hline CGMH & $\begin{array}{l}\text { excellent customer service, polite, } \\
\text { professional, and affordable work } \\
\text {, safe bike tree. }\end{array}$ \\
\hline NMSTG & $\begin{array}{l}\text { excellent food and service and are } \\
\text { amazing service and polite and pro- } \\
\text { fessional . affordable it work out } \\
\text { safe on sun tree! }\end{array}$ \\
\hline $\begin{array}{l}\text { POINTER } \\
\text { (Greedy, } \\
\text { base) }\end{array}$ & $\begin{array}{l}\text { amazing customer service. so polite } \\
\text {, and very professional, and very } \\
\text { affordable . such great work done } \\
\text { at the safe end of a tree. }\end{array}$ \\
\hline $\begin{array}{l}\text { POINTER } \\
\text { (ILBS, } \\
\text { base) }\end{array}$ & $\begin{array}{l}\text { excellent customer service, very po- } \\
\text { lite, and very professional . honest } \\
\text { and affordable pricing . i will defi- } \\
\text { nitely get the work done here for the } \\
\text { safe parts of my tree. }\end{array}$ \\
\hline $\begin{array}{l}\text { POINTER } \\
\text { (Greedy, } \\
\text { Large) }\end{array}$ & $\begin{array}{l}\text { diane provides customers with great } \\
\text { customer service . technician mike } \\
\text { was very polite and helpful . clean } \\
\text { facility, very professional, and al- } \\
\text { ways responsive . quick and afford- } \\
\text { able as well . i had very nice work } \\
\text { done. we have now found someone } \\
\text { safe . thank you big two buck tree } \\
\text { shrub care! }\end{array}$ \\
\hline $\begin{array}{l}\text { Wiki } \\
\text { zero- } \\
\text { shot }\end{array}$ & $\begin{array}{l}\text { customer service should be more po- } \\
\text { lite, and more professional, and } \\
\text { more affordable, and will work in } \\
\text { a safe place under the family tree. }\end{array}$ \\
\hline
\end{tabular}




\begin{tabular}{|c|c|}
\hline Keywords & $\begin{array}{l}\text { hesitate give customers chicken rice } \\
\text { decent list }\end{array}$ \\
\hline ORACLE & $\begin{array}{l}\text { i hesitate to give them the five stars } \\
\text { they deserve because they have a re- } \\
\text { ally small dining area and more cus- } \\
\text { tomers, selfishly, would complicate } \\
\text { things for me . chicken panang is } \\
\text { quite good with a superb brown rice } \\
\text {. decent wine list . after three visits } \\
\text { the wait staff remembered what i like } \\
\text { ( complicated ) and always get the } \\
\text { order right. }\end{array}$ \\
\hline CGMH & $\begin{array}{l}\text { they hesitate to give customers their } \\
\text { chicken fried rice and a decent wine } \\
\text { list. }\end{array}$ \\
\hline NMSTG & $\begin{array}{l}\text { they hesitate to an wonderful time } \\
\text { to give it about a table, love the } \\
\text { customers chicken rice and dishes } \\
\text { seafood and decent at the list. }\end{array}$ \\
\hline $\begin{array}{l}\text { POINTER } \\
\text { (Greedy, } \\
\text { base) }\end{array}$ & $\begin{array}{l}\text { i just did not even hesitate to admit, } \\
\text { i should give credit cards to my cus- } \\
\text { tomers here . the beijing chicken } \\
\text { and fried rice were spot on, a de- } \\
\text { cent side on my favorite list . }\end{array}$ \\
\hline $\begin{array}{l}\text { POINTER } \\
\text { (ILBS, } \\
\text { base) }\end{array}$ & $\begin{array}{l}\text { i don't have to hesitate that they } \\
\text { should give five stars . i will be one } \\
\text { of their repeat customers . like the } \\
\text { basil chicken and basil fried rice, it } \\
\text { was decent on my list. }\end{array}$ \\
\hline $\begin{array}{l}\text { POINTER } \\
\text { (Greedy, } \\
\text { Large) }\end{array}$ & $\begin{array}{l}\text { service is very slow, don't hesitate } \\
\text { to tell manager to give some feed- } \\
\text { backs as their job is to take care of } \\
\text { their customers . had the vegetable } \\
\text { medley soup and chicken . both } \\
\text { were cooked well . the garlic rice } \\
\text { did not have the vegetable and was } \\
\text { fairly decent . they are changing the } \\
\text { flavor and list of menu items. }\end{array}$ \\
\hline $\begin{array}{l}\text { Wiki } \\
\text { zero- } \\
\text { shot }\end{array}$ & $\begin{array}{l}\text { he did not hesitate himself to give it } \\
\text { to his customers, such as chicken } \\
\text {, and steamed rice, a very decent } \\
\text { item on the list. }\end{array}$ \\
\hline
\end{tabular}

\begin{tabular}{|c|c|}
\hline Keyword\$ & $\begin{array}{l}\text { good potential bad maintained re- } \\
\text { placed dirty disgusting }\end{array}$ \\
\hline ORACLE & $\begin{array}{l}\text { has good potential but very bad } \\
\text { maintained . the padding is done } \\
\text {, needs to be replaced, holes every- } \\
\text { where . so are those huge flowers or } \\
\text { what ever those are . ripped . very } \\
\text { dirty too . there was a a very dirty } \\
\text { towel laying on the floor disgusting } \\
\text {. please the city of vegas come and } \\
\text { clean it! }\end{array}$ \\
\hline CGMH & $\begin{array}{l}\text { good potential but bad service. not } \\
\text { maintained . it replaced a dirty box } \\
\text {. disgusting . }\end{array}$ \\
\hline NMSTG & $\begin{array}{l}\text { do a good price . not like the and } \\
\text { potential bad maintained has dis- } \\
\text { gusting . replaced been, dirty and } \\
\text { disgusting. }\end{array}$ \\
\hline $\begin{array}{l}\text { POINTER } \\
\text { (Greedy, } \\
\text { base) }\end{array}$ & $\begin{array}{l}\text { the food was very good . it really has } \\
\text { more potential maybe, but it smells } \\
\text { really bad . its not very well main- } \\
\text { tained either . trash cans were re- } \\
\text { placed only when they were dirty. } \\
\text { the floors were utterly disgusting. }\end{array}$ \\
\hline $\begin{array}{l}\text { POINTER } \\
\text { (ILBS, } \\
\text { base) }\end{array}$ & $\begin{array}{l}\text { the food is really good . this loca- } \\
\text { tion has potential to be pretty bad } \\
\text { and not very well maintained when } \\
\text { it was replaced, its super dirty, just } \\
\text { plain disgusting. }\end{array}$ \\
\hline $\begin{array}{l}\text { PoINTER } \\
\text { (Greedy, } \\
\text { Large) }\end{array}$ & $\begin{array}{l}\text { this gym is not so good . overall it } \\
\text { has a lot of potential for being better } \\
\text { but it is too bad that it is not clean } \\
\text { and un maintained and towels are in } \\
\text { desperate need to be replaced regu- } \\
\text { larly. the floors are very dirty and } \\
\text { the higher floors have become filthy } \\
\text { disgusting when i visited here. }\end{array}$ \\
\hline $\begin{array}{l}\text { Wiki } \\
\text { zero- } \\
\text { shot }\end{array}$ & $\begin{array}{l}\text { it is good it has no potential, and the } \\
\text { bad taste can be maintained until } \\
\text { they are replaced by a dirty, and } \\
\text { disgusting one. }\end{array}$ \\
\hline
\end{tabular}




\begin{tabular}{|c|c|}
\hline Keywords & $\begin{array}{l}\text { love animal style long line expected } \\
\text { quick }\end{array}$ \\
\hline ORACLE & $\begin{array}{l}\text { who doesn } t \text { love in and out . ani- } \\
\text { mal style is a must. long line but } \\
\text { expected, it goes quick anyways so } \\
\text { don t let that discourage you. }\end{array}$ \\
\hline CGMH & $\begin{array}{l}\text { love this place . animal style food } \\
\text { long line than expected for quick }\end{array}$ \\
\hline NMSTG & $\begin{array}{l}\text { love animal chicken. it was style } \\
\text { long a bit so good. the line is it was } \\
\text { even on on a time and we expected } \\
\text { to go but quick. }\end{array}$ \\
\hline $\begin{array}{l}\text { POINTER } \\
\text { (Greedy, } \\
\text { base) }\end{array}$ & $\begin{array}{l}\text { great little breakfast spot. i love } \\
\text { having the double with animal style } \\
\text { fries and protein style etc . have a } \\
\text { super long wait line, but its just as } \\
\text { expected and it always moves pretty } \\
\text { quick too. }\end{array}$ \\
\hline $\begin{array}{l}\text { POINTER } \\
\text { (ILBS, } \\
\text { base) }\end{array}$ & $\begin{array}{l}\text { y all you just gotta love about this } \\
\text { place is the double animal style and } \\
\text { protein style. it was a long line, but } \\
\text { i expected it to be quick. }\end{array}$ \\
\hline $\begin{array}{l}\text { POINTER } \\
\text { (Greedy, } \\
\text { Large) }\end{array}$ & $\begin{array}{l}\text { great burger and good price . i love } \\
\text { that they have non chain locations . } \\
\text { like the animal style fries too . have } \\
\text { to wait long as there is always traf- } \\
\text { fic but the line can be much shorter } \\
\text { than i had expected and they are al- } \\
\text { ways send out pretty quick . very } \\
\text { impressed! }\end{array}$ \\
\hline $\begin{array}{l}\text { Wiki } \\
\text { zero- } \\
\text { shot }\end{array}$ & $\begin{array}{l}\text { he also has love with the animal and } \\
\text { his style, and was long as the finish } \\
\text { line, and was expected to be quick } \\
\text {. }\end{array}$ \\
\hline
\end{tabular}

\begin{tabular}{|c|c|}
\hline Keyword\$ & $\begin{array}{l}\text { great great service happy found close } \\
\text { home }\end{array}$ \\
\hline ORACLE & $\begin{array}{l}\text { great sushi and great service . i m } \\
\text { really happy to have found a good } \\
\text { sushi place so close to home! }\end{array}$ \\
\hline CGMH & $\begin{array}{l}\text { great price and great customer ser- } \\
\text { vice . very happy that i found this } \\
\text { place close to my home. }\end{array}$ \\
\hline NMSTG & $\begin{array}{l}\text { great food and great service . a } \\
\text { happy and found a year in close for } \\
\text { them . keep them home here. }\end{array}$ \\
\hline $\begin{array}{l}\text { POINTER } \\
\text { (Greedy, } \\
\text { base) }\end{array}$ & $\begin{array}{l}\text { amazing food . great quality food } \\
\text { great prices and friendly service } \\
\text { staff. so happy and surprised to have } \\
\text { finally found such a wonderful nail } \\
\text { salon so close to my work and home } \\
\text {. }\end{array}$ \\
\hline $\begin{array}{l}\text { POINTER } \\
\text { (ILBS, } \\
\text { base) }\end{array}$ & $\begin{array}{l}\text { this is just great food . great } \\
\text { food and wonderful service . very } \\
\text { happy to have finally found a chi- } \\
\text { nese restaurant close to my home. }\end{array}$ \\
\hline $\begin{array}{l}\text { POINTER } \\
\text { (Greedy, } \\
\text { Large) }\end{array}$ & $\begin{array}{l}\text { wow . i have been here twice . great } \\
\text { times here. food always has been } \\
\text { great and the customer service was } \\
\text { wonderful . i am very happy that } \\
\text { we finally found our regular pad thai } \\
\text { restaurant that is close to where we } \\
\text { work now and our home . pleasantly } \\
\text { surprised! }\end{array}$ \\
\hline $\begin{array}{l}\text { Wiki } \\
\text { zero- } \\
\text { shot }\end{array}$ & $\begin{array}{l}\text { he was a great teacher and a great } \\
\text { love of the service he was very } \\
\text { happy, and he found himself in the } \\
\text { close to his home. }\end{array}$ \\
\hline
\end{tabular}

\section{Additional Human Evaluation information and Results}

There were 145 judges in all: 5 judges evaluated each pair of outputs to be reasonably robust against spamming. P-values are all $p_{i} 0.00001$ (line 721), computed using 10000 bootstrap replications. Judges were lightly screened by our organization for multiple screening tasks.

We present the additional human evaluation results on POINTER large model vs base model in table 11. In general, for the news dataset the results are mixed. For the yelp dataset, the large model 


\begin{tabular}{rrr|rr|r|r|r|r|r|r}
\hline & \multicolumn{2}{c}{ NIST } & \multicolumn{2}{|c|}{ BLEU } & METEOR & Entropy & Dist & PPL & Avg Len \\
Method & N-2 & N-4 & B-2 & B-4 & & E-4 & D-1 & D-2 & & \\
\hline Greedy (+Wiki) & 3.04 & 3.06 & $13.01 \%$ & $2.51 \%$ & $\mathbf{1 6 . 3 8 \%}$ & 10.22 & $11.10 \%$ & $57.78 \%$ & $\mathbf{5 6 . 7}$ & 31.32 \\
ILBS (+Wiki) & 3.20 & 3.22 & $14.00 \%$ & $2.99 \%$ & $15.71 \%$ & 9.86 & $13.17 \%$ & $61.22 \%$ & 66.4 & 22.59 \\
Greedy (+Wiki,L) & $\mathbf{3 . 2 8}$ & $\mathbf{3 . 3 0}$ & $\mathbf{1 4 . 0 4 \%}$ & $\mathbf{3 . 0 4 \%}$ & $15.90 \%$ & 10.09 & $12.23 \%$ & $60.86 \%$ & $\mathbf{5 4 . 7}$ & 27.99 \\
\hline Wiki zero-shot & 2.80 & 2.82 & $11.38 \%$ & $1.84 \%$ & $15.12 \%$ & 9.73 & $14.33 \%$ & $53.97 \%$ & 62.9 & 20.68 \\
\hline Human & - & - & - & - & - & 10.05 & $11.80 \%$ & $62.44 \%$ & 47.4 & 27.85 \\
\hline
\end{tabular}

Table 9: Additional evaluation results on the News dataset. ILBS denotes beam search. "+Wiki" denotes finetuning on the Wiki-pretrained model. "Human" represents the held-out human reference. "Wiki zero-shot" represents zero-shot generation from the pre-trained model.

\begin{tabular}{rrr|rr|r|r|rr|r|r}
\hline & \multicolumn{2}{c}{ NIST } & \multicolumn{2}{|c|}{ BLEU } & METEOR & Entropy & \multicolumn{2}{|c|}{ Dist } & PPL & Avg Len \\
Method & N-2 & N-4 & B-2 & B-4 & & E-4 & D-1 & D-2 & & \\
\hline Greedy (+Wiki) & 3.27 & 3.30 & $15.63 \%$ & $3.32 \%$ & $16.14 \%$ & 10.64 & $7.51 \%$ & $46.12 \%$ & 71.9 & 48.22 \\
ILBS (+Wiki) & 3.34 & 3.38 & $16.68 \%$ & $3.65 \%$ & $15.57 \%$ & 10.44 & $9.43 \%$ & $50.66 \%$ & 61.0 & 35.18 \\
Large (+Wiki) & $\mathbf{3 . 4 9}$ & $\mathbf{3 . 5 3}$ & $\mathbf{1 6 . 7 8 \%}$ & $\mathbf{3 . 7 9 \%}$ & $16.69 \%$ & 10.56 & $6.94 \%$ & $41.2 \%$ & $\mathbf{5 5 . 5}$ & 48.05 \\
\hline Wiki zero-shot & 0.86 & 0.87 & $8.56 \%$ & $1.30 \%$ & $12.85 \%$ & 9.90 & $10.09 \%$ & $41.97 \%$ & 62.9 & 26.80 \\
\hline Human & - & - & - & - & - & 10.70 & $10.67 \%$ & $52.57 \%$ & 55.4 & 50.36 \\
\hline
\end{tabular}

Table 10: Additional evaluation results on the Yelp dataset. ILBS denotes beam search. "+Wiki" denotes finetuning on the Wiki-pretrained model. "Human" represents the held-out human reference. "Wiki zero-shot" represents zero-shot generation from the pre-trained model.

\begin{tabular}{|c|c|c|c|c|c|c|c|c|}
\hline \multicolumn{9}{|c|}{ Informativeness: $A$ and $B$, which is more semantically meaningful and consistent? } \\
\hline \multicolumn{2}{|l|}{ System A } & Neutral | & ystem B & \multicolumn{2}{|c|}{ System A } & \multirow{2}{*}{$\frac{\text { Neutral }}{26.6 \%}$} & \multirow{2}{*}{\multicolumn{2}{|c|}{$\frac{\text { System B }}{\text { PoInTER(base) *** }}$}} \\
\hline POINTER(large) & $35.4 \%$ & \begin{tabular}{l|l}
$27.7 \%$ & $\mathbf{3 6 . 9} \%$
\end{tabular} & POINTER(base) & POINTER(large) & $41.4 \%$ & & & \\
\hline POINTER(large) & $20.3 \%$ & $22.7 \% \quad \mathbf{5 7 . 1} \%$ & Human $* * *$ & POINTER(large) & $27.2 \%$ & $24.4 \%$ & $48.5 \%$ & Human $* * *$ \\
\hline \multicolumn{2}{|l|}{ System A } & Neutral | & System B & \multicolumn{2}{|c|}{ System A } & Neutral & & System B \\
\hline POINTER(large) & $38.4 \%$ & \begin{tabular}{l|l}
$28.5 \%$ & $33.2 \%$
\end{tabular} & PoInTER(base) & POINTER(large) & $41.1 \%$ & $28.1 \%$ & $30.8 \%$ & POINTER(base) $* * *$ \\
\hline POINTER(large) & $16.7 \%$ & \begin{tabular}{l|l}
$15.8 \%$ & $\mathbf{6 7 . 5} \%$
\end{tabular} & Human $* * *$ & POINTER(large) & $27.1 \%$ & $21.9 \%$ & $\mathbf{5 1 . 1} \%$ & Human $* * *$ \\
\hline POINTER(large) & $32.1 \%$ & \begin{tabular}{l|l}
$27.6 \%$ & $\mathbf{4 0 . 4} \%$
\end{tabular} & POINTER(base) & POINTER(large) & $41.6 \%$ & $25.0 \%$ & $33.4 \%$ & POINTER(base) $* * *$ \\
\hline POINTER(large) & $31.9 \%$ & \begin{tabular}{l|l|}
$17.1 \%$ & $\mathbf{5 1 . 0} \%$ \\
\end{tabular} & Human $* * *$ & POINTER(large) & $35.9 \%$ & $14.7 \%$ & $49.4 \%$ & Human $* * *$ \\
\hline
\end{tabular}

Table 11: Human Evaluation on two datasets for semantic consistency, fluency and informativeness, showing preferences (\%) for our Pointer(large) model vis-a-vis Pointer(base) model and real human responses. Numbers in bold indicate the most preferred systems. Significant differences $(\mathrm{p} \leq 0.001)$ are indicated as $* * *$.

wins with a large margin. All results are still far away from the human oracle in all three aspects.

\section{E Additional Automatic Evaluation Results}

We provide the full evaluation result data including Wikipedia zero-shot learning results in Table 9 and Table 10. Note that zero-shot generations from Wikipedia pre-trained model yield the lowest perplexity, presumably because the Wikipedia dataset is large enough so that the model trained on it can learn language variability, thus delivering fluent generated results.

\section{F Inference Details}

During inference time, we use a decaying schedule to discourage the model from generating noninteresting tokens, including [NOI] and some other special tokens, punctuation and stop words. To do this, we use a decay multiplier $\eta$ on the logits of 
these tokens before computing the softmax. The $\eta$ is set to be $\eta=\min (0.5+\lambda * s)$, where $s$ is the current stage and $\lambda$ is an annealing hyper-parameter.

In most of the experiments, $\lambda$ is set at 0.5

\section{G Human Evaluation Template}

See Figure 2 for human evaluation template 


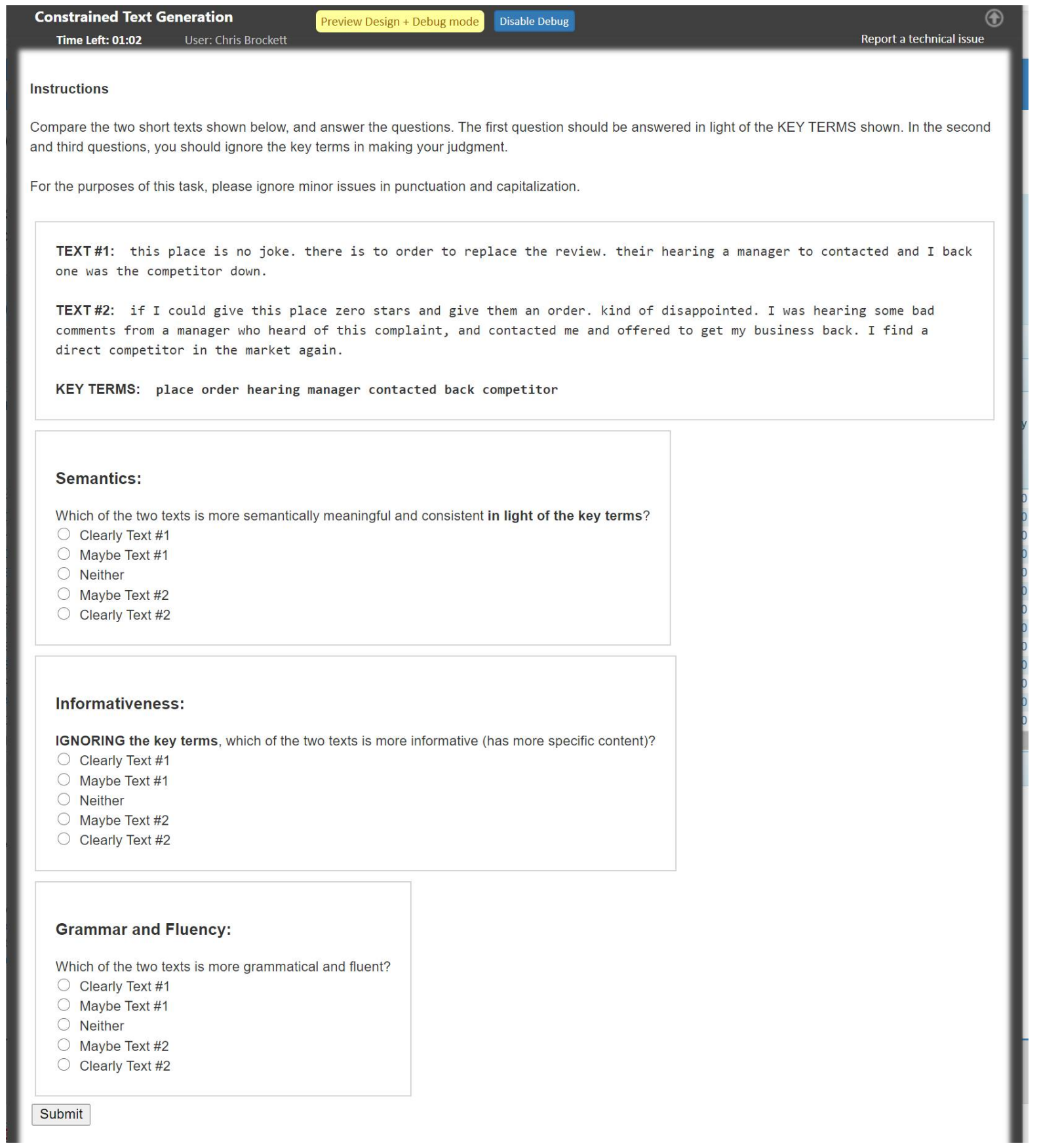

Figure 2: Human evaluation template. 